\title{
Fluorescent Light Energy (FLE) Acts on Mitochondrial Physiology Improving Wound Healing
}

\author{
Letizia Ferroni $\left.{ }^{1,2,+}{ }^{(}\right)$, Michela Zago ${ }^{3,+}$, Simone Patergnani ${ }^{1,4}{ }^{\oplus}$, Shannon E. Campbell ${ }^{3}$, \\ Lise Hébert ${ }^{3}$, Michael Nielsen ${ }^{5}$, Carlotta Scarpa ${ }^{6}$, Franco Bassetto ${ }^{6}$, Paolo Pinton ${ }^{1,4}$ and \\ Barbara Zavan 1,2, *(D) \\ 1 Maria Cecilia Hospital, GVM Care \& Research, 48033 Cotignola (RA), Italy \\ Department of Medical Sciences, University of Ferrara, 44121 Ferrara, Italy \\ Klox Technologies Inc., 275 Armand-Frappier Blvd, Laval, QC H7V 4A7 Canada \\ 4 Department of Morphology, Surgery and Experimental Medicine, Section of Pathology, Oncology and \\ Experimental Biology, Laboratory for Technologies of Advanced Therapies (LTTA), University of Ferrara, \\ 44121 Ferrara, Italy \\ 5 Klox Technologies Inc., Denmark ApS, Borupvang 5C, 2750 Ballerup, Denmark \\ 6 Clinic of Plastic and Reconstructive Surgery, Department of Neurosciences, University Hospital of Padova, \\ 35131 Padova, Italy \\ * Correspondence: barbara.zavan@unife.it \\ $\dagger$ co first author.
}

Received: 24 December 2019; Accepted: 6 February 2020; Published: 18 February 2020

check for updates

\begin{abstract}
Fluorescent light energy (FLE) has been used to treat various injured tissues in a nonpharmacological and non- thermal fashion. It was applied to stimulate cell proliferation, accelerate healing in chronic and acute wounds, and reduce pain and inflammation. FLE has been shown to reduce pro-inflammatory cytokines while promoting an environment conducive to healing. A possible mechanism of action of FLE is linked to regulation of mitochondrial homeostasis. This work aims to investigate the effect of FLE on mitochondrial homeostasis in an in vitro model of inflammation. Confocal microscopy and gene expression profiling were performed on cultures of inflamed human dermal fibroblasts treated with either direct light from a multi-LED lamp, or FLE from either an amorphous gel or sheet hydrogel matrix. Assessment using confocal microscopy revealed mitochondrial fragmentation in inflamed cells, likely due to exposure to inflammatory cytokines, however, mitochondrial networks were restored to normal 24-h after treatment with FLE. Moreover, gene expression analysis found that treatment with FLE resulted in upregulation of uncoupling protein 1 (UCP1) and carnitine palmitoyltransferase 1B (CPT1B) genes, which encode proteins favoring mitochondrial ATP production through oxidative phosphorylation and lipid $\beta$-oxidation, respectively. These observations demonstrate a beneficial effect of FLE on mitochondrial homeostasis in inflamed cells.
\end{abstract}

Keywords: fluorescent light energy; inflammation; wound healing; mitochondria; gene expression; mitochondrial dynamics; fluorescence

\section{Introduction}

Wound healing follows a series of complex overlapping processes that leads to closure of the wound and restoration of the epithelial layer, including hemostasis, inflammation, cell proliferation and tissue remodeling [1].

After an injury to skin, the exposed sub-endothelium, collagen and tissue factors activate platelet aggregation to form a clot (hemostasis). Next, neutrophils appear at the site of injury to remove debris and bacteria, promoting an environment for wound healing. Then, macrophages 
accumulate in the inflammatory phase facilitating phagocytosis of bacteria and damaged tissue. In acute wounds, inflammation is fast and short-lived (typically 3-5 days). The inflammatory cells orchestrate the inflammatory process and facilitate initiation of the reconstructive phase of healing. Lamentably, in chronic non-healing wounds the inflammatory cytokines are in excess, and the resultant pro-inflammatory environment causes degradation of growth factors and extracellular matrix (ECM) proteins [2]. In acute wound, the proliferative phase follows the inflammatory phase, and is characterized by accumulation of fibroblasts and production of connective tissue. Fibroblasts play a critical role in supporting normal wound healing due to their involvement in several key processes, including breakdown of fibrin clot, creation of new ECM and collagen structures, and contraction of the wound [3]. Finally, during the tissue remodeling phase, collagen bundles are reorganized, restoring epidermal barrier function and skin integrity. Overall, the healing process involves numerous cellular and biosynthetic processes, which all require energy in the form of adenosine triphosphate (ATP), as well as amino acids, and other precursor molecules to replace damaged tissue [4-6].

Mitochondria are the key organelles responsible for ATP production in human cells, generating energy through oxidative phosphorylation [7]. The mitochondrial respiratory chain consists of a five-subunit complex (I-V) through which electrons are exchanged at increasing reduction potentials, leading to production of ATP [8]. Besides production of ATP for cellular energy, mitochondria also generate reactive oxygen species (ROS) as a by-product of ATP formation [9]. The role of ROS in wound healing is complex. ROS contributes to the oxidative burst that kills bacteria, and acts as a cell signal to increase cell proliferation, which is vital in wound repair [10]. However, ROS are also very potent molecules and can cause damage to DNA, lipids and proteins [11]. Excessive levels of ROS have been associated with chronic wounds, resulting in tissue damage, excessive inflammation, and delayed healing $[12,13]$. Furthermore, high levels of oxidative stress impact on mitochondrial morphology and positioning within cells, causing mitochondria to cluster around the nucleus as a protective strategy [14,15].

Generally, mitochondria undergo a constant process of fission and fusion where they join and subsequently split back into separate entities. This process is thought to be a housekeeping effect to ensure that mitochondria stay as efficient as possible [16]. The position of mitochondria within cells is dynamic based on localized signals or energy balance. For instance, during cell migration mitochondria will locate close to the migrating cell edges where rapid reorganization of the cytoskeleton filaments requires more ATP for energy [17-19].

Photobiomodulation (PBM) is the term used to describe the application of low-level light energy to induce tissue regeneration or to protect tissue that is injured or degenerating [20-22]. PBM has been shown to be effective in accelerating healing in chronic and acute wounds [23,24], as well as reducing pain and inflammation in several conditions [25-27]. Moreover, PBM reverses toxic effects of neurotoxins, stimulates stem cell proliferation, and displays therapeutic effects in reducing myocardial ischemia reperfusion related to myocardial injury [28,29].

One of the most well-studied mechanisms of PBM is the ability to interact with endogenous chromophores in tissue that absorb photons (light). The most studied endogenous chromophore is complex IV of the mitochondrial respiratory chain, cytochrome c oxidase (CCO). Studies have shown that the activation spectrum of CCO runs from yellow through to red wavelengths ( 570-910 $\mathrm{nm})$. Absorption of photons by CCO initiates a biochemical cascade that increases ATP and ROS generation within the electron transport chain [30-33]. Additionally, evidence suggests that one possible mechanism of action of photons on the wound healing process is regulation of the homeostatic balance between mitochondrial fusion and fission [34]. Mitochondria fusion helps reduce mitochondrial stress by mixing the contents of partially damaged mitochondria. Fission allows the creation of new mitochondria, but it also enables the removal of damaged mitochondria [35].

A form of PBM is fluorescent light energy (FLE) that acts as a vehicle to induce biomodulation. To generate fluorescence, specialized chromophores (light absorbing molecules) are employed to translate light energy into a low-energy emission of fluorescence through a mechanism known as 
stokes shift [36]. FLE is a unique form of photobiomodulation that has been demonstrated to advance healing of both acute and chronic wounds $[37,38]$. Studies have demonstrated that acute incisional wounds have reduced inflammation, as well as more physiologic re-epithelization and collagen remodeling resulting in better quality and less visible scars [25-27,39]. Additionally, patients with hard-to-heal chronic ulcers experienced accelerated healing and improved quality of life [23]. These studies have documented the safety and efficacy of FLE in a variety of impaired skin conditions [40]. The beneficial impact of FLE on inflammation has been well documented, with improved inflammatory profiles observed in clinical trials for wound healing [37], acne treatment [41], and management of rosacea [42], as well as in canine pyoderma [43] and mechanistic in vitro studies [36]. These studies have demonstrated that FLE has the ability to reduce pro-inflammatory cytokines produced by human dermal fibroblasts (HDFs), as well as increase levels of some anti-inflammatory cytokines, which have a beneficial effect in the treatment of chronic wounds that are stuck in an inflammatory feedback loop [36].

The effect of FLE on mitochondrial homeostasis is still largely unknown. We tested the effect of FLE on an in vitro model of inflammation. Cultures of normal HDFs were stimulated with a cocktail of pro-inflammatory cytokines to create inflamed cells. These inflamed cells were treated with either direct light (photobiomodulation) or FLE. Two forms of photoconverter substrates were tested: an amorphous gel (FLE-Gel) or sheet hydrogel matrix (FLE-Matrix). Variations in the mitochondrial network were analyzed with confocal microscopy. Expression profiles of genes related to mitochondrial dynamic, biogenesis and function were assessed by PCR gene array. Confocal microscopy showed the presence of mitochondrial fragmentation due to exposure to the inflammatory cytokines, but a restoration of the mitochondrial network $24 \mathrm{~h}$ post-treatments was observed, in particular in cultures treated with FLE. The gene expression analysis showed that treatment with FLE-Matrix upregulated UCP1 and CPT1B genes, which encode proteins that favor ATP production through oxidative phosphorylation and lipid $\beta$-oxidation, respectively. Treatment with FLE-Gel upregulated SLC25A31 gene, linked to cytosolic adenosine diphosphate (ADP) balance. These observations, together with the already known capacity of photobiomodulation to stimulate cytochrome c oxidase, show a beneficial effect of FLE in the treatment of inflamed wounds.

\section{Experimental Section}

\subsection{Cell Culture Preparation}

Normal human dermal fibroblasts (HDFs; PCS-201-012, American Type Culture Collection (ATCC, Manassas, VA, USA) were cultured at $37^{\circ} \mathrm{C}$ and $5 \% \mathrm{CO}_{2}$ in Fibroblast Basal Medium (phenol red-free; ATCC, Manassas, VA, USA) supplemented with Fibroblast Growth Kit-Low serum (ATCC, Manassas, VA, USA). The culture process was performed seeding HDFs in 6-well plastic plates at a density of $10 \times 10^{4}$ cell/well. Healthy cells were incubated in basal medium for the duration of the experiment.

Inflamed cells (including all treatment groups) were incubated for $5-6 \mathrm{~h}$ in basal medium prior to inducing an in vitro inflammatory state with an 18-h incubation in an inflammatory cocktail comprising $20 \mathrm{ng} / \mathrm{mL}$ each of pro-inflammatory cytokines recombinant human tumor necrosis factor alpha (TNF- $\alpha$; Miltenyi Biotec S.r.l., Bologna, Italy) and recombinant human Interleukin-1 beta (IL-1 $\beta$; Miltenyi Biotec S.r.l.).

For all treatment groups (Light, FLE-Gel, and FLE-Matrix), after the 18-h incubation in the inflammatory cocktail, the media was replaced with Phosphate Buffer Saline (PBS; Euroclone S.p.A., Italy) for the illumination procedure (5-min) to minimize scattering or other interference with the light. After the illumination, the PBS was replaced with fresh media containing the TNF $\alpha / \mathrm{IL}-1 \beta$ inflammatory cocktail to continue with the inflammatory stimulus. Untreated inflamed HDFs were also placed in PBS for $5 \mathrm{~min}$ (to mimic the treatment conditions), and then were incubated in fresh media containing inflammatory cockail for the duration of the experiment. 
Mitochondrial morphology was analyzed at 30-min and 24-h post-treatment for all groups, and gene expression profile was investigated at 6-h post-treatment.

\subsection{Fluorescent Light Energy (FLE) Systems}

FLE Systems consist of a multi-LED lamp (KT-L lamp, Klox Technologies Inc., Laval, QC, Canada) and a topical photoconverter substrate in the form of an amorphous gel (FLE-Gel) or sheet hydrogel matrix (FLE-Matrix) (LumiHeal ${ }^{\mathrm{TM}}$ Gel and LumiHeal ${ }^{\mathrm{TM}}$ Matrix, Klox Technologies Inc., Laval, QC, Canada). The multi-LED lamp delivers non-coherent light between $400-520 \mathrm{~nm}$ with a peak at app. $447 \mathrm{~nm}$ and a power density between $110-150 \mathrm{~mW} / \mathrm{cm}^{2}$ at a distance of $5 \mathrm{~cm}$ from the light-emitting diodes (LEDs). The lamp is equipped with a 5 -min timer and a distance indicator. FLE photoconverters contain a chromophore, embedded within the gel or matrix, which can absorb some of the photons from the multi-LED lamp, and emit FLE in the range of approximately 510-700 nm. Thus cells treated with FLE receive a combination of direct light from the multi-LED lamp plus FLE emitted from the Gel or Matrix photoconverter, for delivery of a full spectral range between $400-700 \mathrm{~nm}$. Of note, a dose response for FLE may be observed by assessing FLE-Gel compared with FLE-Matrix, as FLE-Gel generates $0.1-0.2 \mathrm{~J} / \mathrm{cm}^{2}$ of fluorescence $(\sim 510-700 \mathrm{~nm})$ whereas FLE-Matrix generates $0.2-0.7 \mathrm{~J} / \mathrm{cm}^{2}$.

\subsection{Fluorescence Light Energy (FLE) Protocols}

Three treatment conditions were tested in order to study the impact of FLE on mitochondrial morphology and gene expression. Light-treated cells received a 5-min illumination with the multi-LED lamp placed $5 \mathrm{~cm}$ from the bottom of the plate, without the presence of a topical photoconverter. For FLE-treated cells (FLE-Gel or FLE-Matrix) the topical photoconverters were placed under the 6-well plate, not in direct contact with cells, and the multi-LED lamp was placed at $5 \mathrm{~cm}$ from the bottom of the plate. Light and FLE are transmitted unchanged through the plastic bottom of the plate, thus contact with the cells is not required to induce their effects. The illumination duration was 5-min for all treatment groups. Each of the following groups were tested:

(a) Healthy: HDFs maintained in basal medium (no inflammatory cocktail or illumination).

(b) Inflamed: HDFs incubated in TNF $\alpha / \mathrm{IL}-1 \beta$ inflammatory cocktail.

(c) Light: Inflamed HDFs illuminated for 5-min with only the multi-LED lamp (no FLE).

(d) Gel: Inflamed HDFs illuminated for 5-min with the FLE-Gel system consisting of the multi-LED lamp and topical photoconverter amorphous gel (LumiHeal Gel, Klox Technologies Inc., Laval, QC, Canada).

(e) Matrix: Inflamed HDFs illuminated for 5-min with the FLE-Matrix system consisting of the multi-LED lamp and topical photoconverter sheet hydrogel matrix (LumiHeal Matrix, Klox Technologies Inc., Laval, QC, Canada).

Healthy HDFs were considered as the control group of the experiment.

\subsection{Mitochondrial Morphology}

Cells were seeded in coverslips 24-mm in diameter and allowed to grow to a confluence of 50-60\%. After treatments, cells were fixed with $4 \%$ paraformaldehyde solution (Sigma-Aldrich, USA) and washed three-times. Next, cells were permeabilized with a solution of $0.1 \%$ triton $\mathrm{x}-100$ (Sigma-Aldrich, USA), for $10 \mathrm{~min}$ at room-temperature (RT) on a plate-shaker. After three-washes, unspecific sites were blocked with a solution of $2 \%$ bovine serum albumin (Sigma-Aldrich, USA) supplemented of $0.01 \%$ triton $\mathrm{x}-100$ for $45 \mathrm{~min}$. at RT with agitation. Cells were next incubated with a primary antibody against TOM20 (mitochondrial marker of the inner membrane) (BD, USA) diluted 1:100 over-night at $4{ }^{\circ} \mathrm{C}$. The next day, cells were washed with three washes of $10 \mathrm{~min}$ each with agitation at RT and next incubated with a specific secondary fluorescent antibody Alexa Fluor 488 (Thermo Fisher Scientific, Waltham, USA) diluted 1:1000 in the dark for $45 \mathrm{~min}$ at RT with agitation. Cells were acquired in z-stacks of 51 planes at $0.2 \mu \mathrm{m}$ each at Nikon A1 confocal microscope equipped with a 
$63 \mathrm{X}$ objective. Images obtained were deconvolved to remove blurred signal and 3D reconstructed. The mitochondrial network was then quantified by using the 3D-object counter available in software Fiji (http://fiji.sc/wiki/index.php/Fiji accessed on 29 April 2017) that allow to measure the total object (mitochondria) volume and the number of total objects (mitochondria) per each cell. The mean volume of single mitochondria was calculated by divide the total mitochondria volume with the number of total mitochondria. For each condition, at least 20 cells were analyzed. Data are presented as mean \pm SD. Multi comparison statistical analyses were performed by using one-way ANOVA. T test was to perform all pairwise comparisons between group means. Calculated mean $\pm \mathrm{SD}$ are reported in figure legends.

\subsection{Total RNA Isolation and PCR Array Profile}

As previously described [44], total RNA was extracted by the RNeasy Mini Kit (Qiagen, Hilden Germany) which includes DNase digestion using the RNase-Free DNase Set (Qiagen). For each sample, 500 ng of total RNA were reverse transcribed with RT $^{2}$ First Strand Kit (Qiagen) in SimpliAmp Thermal Cycler (Thermo Fisher Scientific) following the manufacture procedures. Then, the RT ${ }^{2}$ Profiler PCR Array Human Mitochondrial Energy Metabolism (Qiagen) and RT ${ }^{2}$ Profiler PCR Array Human Mitochondria (Qiagen) in StepOne Plus Real-Time PCR System (Thermo Fisher Scientific) were performed. The amplification protocol included the activation at $95^{\circ} \mathrm{C}$ for $10 \mathrm{~min}$, followed by 40 cycles of denaturation at $95^{\circ} \mathrm{C}$ for $15 \mathrm{~s}$, and elongation at $60^{\circ} \mathrm{C}$ for $1 \mathrm{~min}$. The $2 \Delta \Delta \mathrm{CT}$ method was used to determine the relative expression of target genes. Cycle threshold $(\mathrm{Ct})$ values of target genes were normalized to the geometric mean $\mathrm{Ct}$ values of five housekeeping genes (ACTB: actin, beta; B2M: beta-2-microglobulin; GAPDH: glyceraldehyde-3-phosphate dehydrogenase; HPRT1: hypoxanthine phosphoribosyl transferase 1; RPLP0: ribosomal protein, large, P0). For each target gene, the average of three normalized expression levels were calculated, and $p$ values were calculated using Student's $\mathrm{t}$-test based on $2 \Delta \mathrm{CT}$ values for each gene in the test group compared to the control group. Statistical significance was set at $p<0.05$. Results were reported as fold regulation of target genes in test group compared with control group.

\section{Results}

\subsection{Mitochondrial Morphology Analysis}

Analysis of the mitochondrial morphology was performed in all HDF conditions: (a) healthy HDFs in normal media (Healthy), (b) inflamed HDFs in TNF $\alpha / \mathrm{IL}-1 \beta$ inflammatory cocktail (Inflamed), (c) inflamed HDFs treated with light alone (Light), (d) inflamed HDFs treated with FLE-Gel (Gel), and (e) inflamed HDFs treated with FLE-Matrix (Matrix). Importantly, incubation of HDFs with the TNF $\alpha / \mathrm{IL}-1 \beta$ inflammatory cocktail led to fragmentation of the mitochondrial network (Figure 1). A global reduction of mitochondrial volume per cell was observed in Inflamed HDFs (Figure 1a) accompanied by an increase in the number of mitochondria per cell, and a reduction of individual mitochondrion volume (Figure 1b,c, respectively). Predictably, greater exacerbation of the mitochondria was observed in Inflamed HDFs at $24 \mathrm{~h}$ compare to $30 \mathrm{~min}$, due to the continued exposure to the inflammatory cocktail (Figure 2).

Interestingly, when inflamed cells were exposed to either direct light (Light) or FLE (Gel or Matrix) the mitochondrial network showed preliminary signs of improvement at 30-min post-treatment, with some statistical differences already observed in FLE-treated cells. This improvement occurred despite the continued exposure to the TNF $\alpha / \mathrm{IL}-1 \beta$ inflammatory cocktail. Mitochondrial number per cell in Inflamed HDFs was $51 \pm 13$, compared with $41 \pm 11$ in FLE-Gel HDFs $(p=0.019)$ and $41 \pm 12$ in FLE Matrix HDFs $(p=0.0148)$. Similarly, individual mitochondrion volume was $12.8 \pm 4.13 \mu \mathrm{m}^{3}$ in Inflamed HDFs, compared with $18.3 \pm 6.5 \mu \mathrm{m}^{3}(p=0.0185)$ and 18.6 $\pm 6.3 \mu \mathrm{m}^{3}(p=0.0156)$ in FLE-Gel and FLE-Matrix HDFs, respectively. Taken together, this data indicates that mitochondria in FLE-treated cells have shifted their mitochondrial network into a more fused state, with larger mitochondria that 
are fewer in number. While the difference in the volume of the entire mitochondrial network per cell did not reach significance between Inflamed and FLE-treated cells at the $30 \mathrm{~min}$ time point, it is interesting to note that while both Inflamed and Light-treated HDFs are different from Healthy HDFs $\left(648 \pm 237 \mu \mathrm{m}^{3}\right.$ and $639 \pm 273 \mu \mathrm{m}^{3}$ vs. $840 \pm 153 \mu \mathrm{m}^{3}, p=0.0103$ and 0.0093 respectively), neither of the FLE-treated groups were significantly different from Healthy HDFs, suggesting they are already starting to recover from the inflamed state.

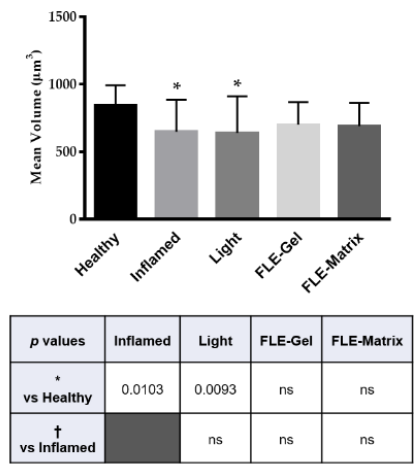

(a)
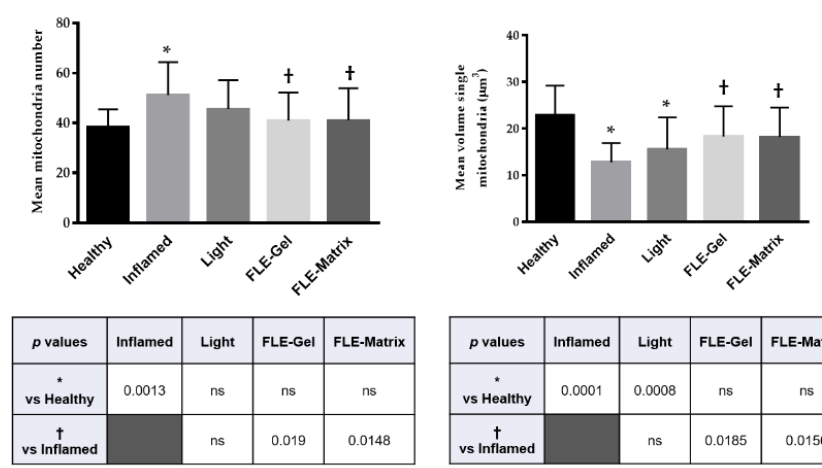

(b)

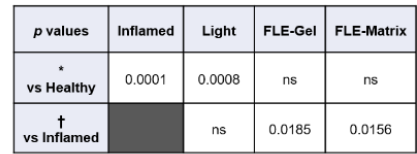

(c)
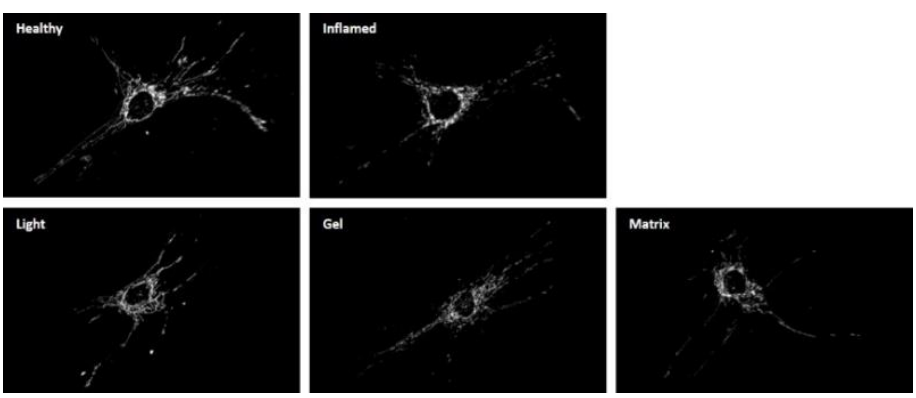

(d)

Figure 1. Quantification of mitochondrial network 30-min post-treatment. After deconvolution, images were $3 \mathrm{D}$ reconstructed and the mitochondrial network was evaluated by automated estimation of (a) volume of the entire mitochondrial network per single cell, (b) number of mitochondria per single cell, and (c) volume of single mitochondrion. Data are expressed as mean \pm SD. Multi comparison statistical analysis were performed by using one-way analysis of variance (ANOVA). T tests were performed on all pairwise comparisons between group means. ${ }^{*} p<0.05$ from Healthy human dermal fibroblasts (HDFs), $+<0.05$ from Inflamed human dermal fibroblasts (HDFs), ns = not significant. (d) Representative images. FLE: fluorescent light energy.

In order to verify this possibility, the healthiness of the mitochondrial network was analyzed for a longer period of time. At 24-h post-treatment Inflamed HDFs treated with FLE had completely recovered to healthy-HDF mitochondrial morphology, while cells treated with Light were only partially recovered (Figure 2). All observed aspects of the mitochondrial networks were significantly improved in FLE-treated cells compared with Inflamed HDFs. Volume of the entire mitochondrial network per cell was $802 \pm 133 \mu \mathrm{m}^{3}$ in FLE-Gel HDFs, and $910 \pm 159 \mu \mathrm{m}^{3}$ in FLE-Matrix, compared with $523 \pm 301 \mu \mathrm{m}^{3}$ in Inflamed HDFs ( $p=0.0018$ and 0.0001 , respectively). Values for the FLE-treated cells were not significantly different from Healthy HDFs $\left(845 \pm 205 \mu \mathrm{m}^{3}\right)$. Additionally, the count of mitochondria number per cell for Inflamed HDFs was $57 \pm 16$ compared with $48 \pm 14$ and $39 \pm 9$ for FLE-Gel and FLE-Matrix ( $p=0.0016$ and $p=0.0001$, respectively), and individual mitochondrion volume was $9.5 \pm 3.5 \mu \mathrm{m}^{3}$ in Inflamed HDFs compared with $20 \pm 5.6 \mu \mathrm{m}^{3}$ and $24 \pm 7.5 \mu \mathrm{m}^{3}$ for FLE-Gel and FLE-Matrix ( $p=0.0016$ and $p=0.0001$, respectively). By comparison, Light-treatment had induced 
some recovery, and was no longer significantly different from healthy HDFs in any parameter. However, it was only significantly improved compared with Inflamed cells in the individual mitochondrion volume (10 \pm 3 vs. $16 \pm 4 \mu \mathrm{m}^{3}$ for Inflamed vs. Light respectively, $\left.p=0.0104\right)$.

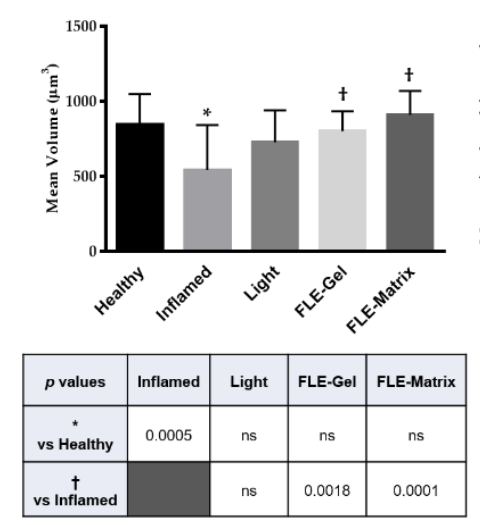

(a)

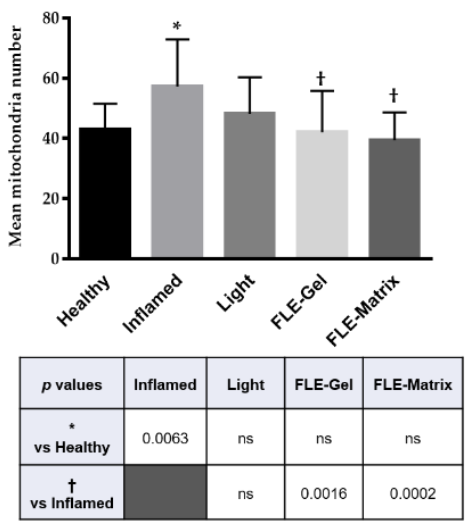

(b)

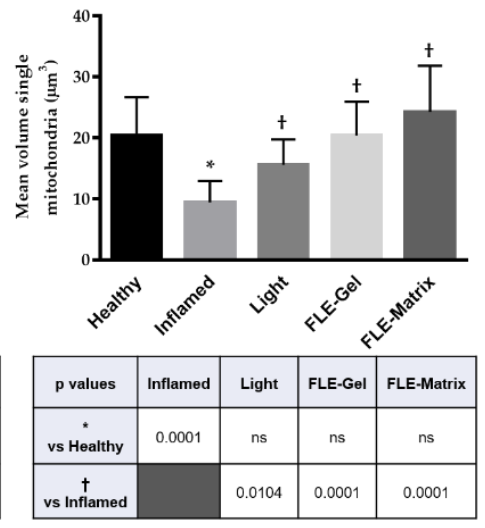

(c)
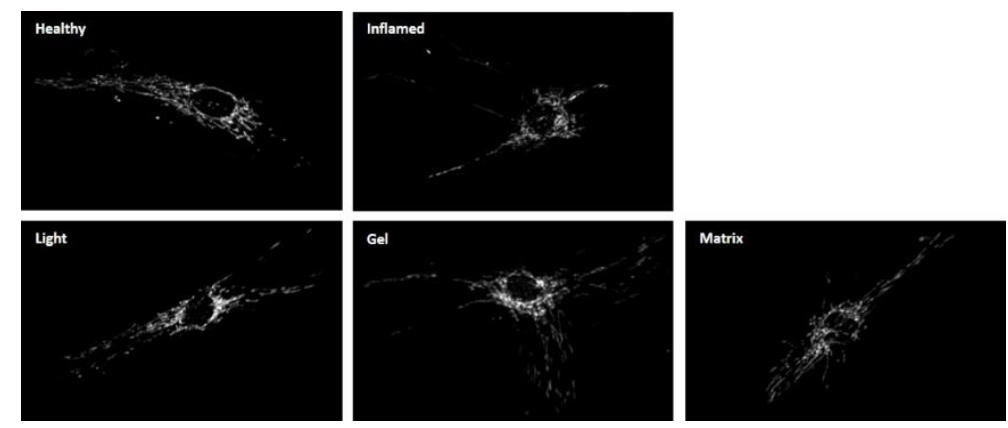

(d)

Figure 2. Quantification of mitochondrial network 24-h post-treatment. After deconvolution, images were $3 \mathrm{D}$ reconstructed and the mitochondrial network was evaluated by automated estimation of (a) volume of the entire mitochondrial network per single cell, (b) number of mitochondria per single cell, and (c) volume of single mitochondrion. Data are expressed as mean \pm SD. Multi comparison statistical analysis were performed by using one-way analysis of variance (ANOVA). $\mathrm{T}$ test was to perform all pairwise comparisons between group means. ${ }^{*} p<0.05$ from Healthy human dermal fibroblasts (HDFs), $+<0.05$ from Inflamed human dermal fibroblasts (HDFs), ns = not significant. (d) Representative images. FLE: fluorescent light energy.

In Figure 3, radial graphs provide a visual representation of the ability of FLE to fully rescue the mitochondrial dynamics of inflamed HDFs. All values are indexed to the healthy values for volume of the mitochondrial network per cell (top, Mitochondria Volume per cell, MV/c), number of mitochondria per cell (bottom right, Mitochondria Number per cell, MN/c) and individual mitochondrion volume (bottom left, Individual Mitochondrion Volume, IMV). The green line represents the Healthy condition, and the red line indicates the Inflamed status. At both the 30-min (Figure 2a) and 24-h (Figure 2b) post-treatment time points, it is apparent that Light-treated cells (blue lines) are shifting away from the Inflamed state towards the Healthy condition. A noticeably greater recovery is visible in FLE-treated cells (FLE-Gel in yellow and FLE-Matrix in orange) at 30-min post-treatment, where both have already progressed beyond the Light cells toward the Healthy condition. Impressively, by 24-h post-treatment FLE-treated cells are completely recovered, even overlapping with the Healthy condition. 


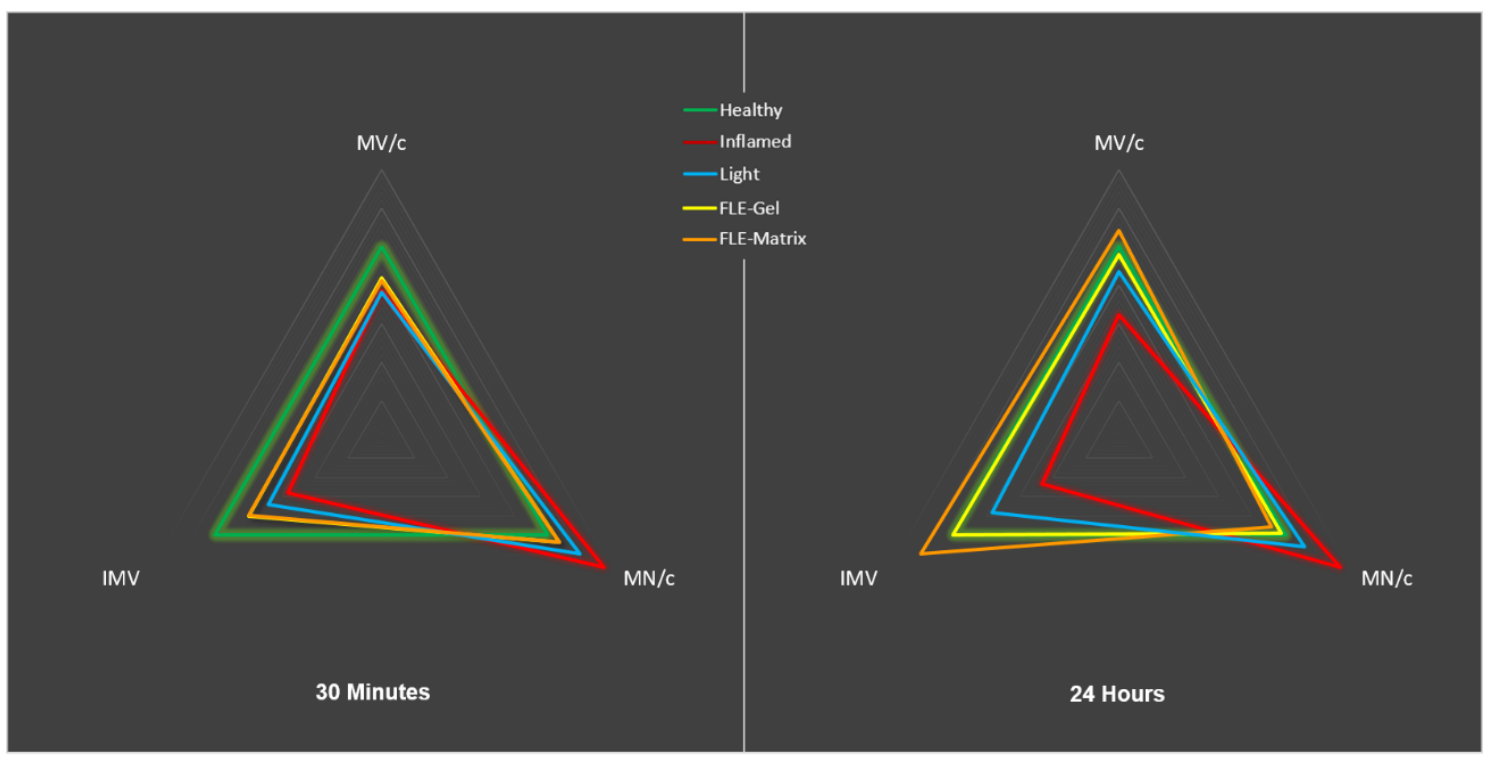

(a)

(b)

Figure 3. Radial graphs depicting relative changes in mitochondrial networks at (a) 30-min and (b) 24-h post-treatment. All parameters are indexed to Healthy-HDFs (green). Inflamed-HDFs are depicted in red, Light-treated HDFs in blue, FLE-Gel in yellow, and FLE-Matrix in orange. At time point 30-min the FLE-Gel (yellow) and FLE-Matrix (Orange) overlap, and at time point 24-h the Healthy (green) and FLE-Gel (yellow) overlap. $M V / c=$ Mitochondria Volume per cell; $M N / c=$ Mitochondria Number per cell; $I M V=$ Individual Mitochondrion Volume.

\subsection{PCR Array Gene Expression Profile}

In order to investigate the biological impact of the treatments on mitochondrial dynamic and function, mitochondrial gene expression was probed with two different real-time PCR array, measured 6-h post-treatment.

Table 1 presents data on the expression of 84 key genes associated with mitochondrial respiration, including genes encoding components of the electron transport chain and oxidative phosphorylation complexes. Oxidation of Nicotinamide adenine dinucleotide (NADH) and flavin adenine dinucleotide (FADH2), key metabolites in glycolysis and the citric acid cycle, occurs within a series of four protein complexes embedded in the inner mitochondrial membrane: $\mathrm{NADH}$-coenzyme $\mathrm{Q}$ reductase, succinate-coenzyme $Q$ reductase, coenzyme $Q$-cytochrome c reductase, and CCO. The free energy generated from these processes drives oxidative phosphorylation and ATP synthesis via a fifth protein complex (ATP synthase) [45].

Table 1. Human Mitochondrial Energy Metabolism PCR array.

\begin{tabular}{ccccccccc}
\hline Gene & $\begin{array}{c}\text { Inflamed vs. } \\
\text { Healthy }\end{array}$ & $p$ Value & $\begin{array}{c}\text { Light vs. } \\
\text { Inflamed }\end{array}$ & $p$ Value & $\begin{array}{c}\text { FLE-Gel vs. } \\
\text { Inflamed }\end{array}$ & $p$ Value & $\begin{array}{c}\text { FLE-Matrix } \\
\text { vs. Inflamed }\end{array}$ & $p$ Value \\
\hline ATP12A & 1.08 & 0.3024 & 1.31 & 0.5219 & -1.11 & 0.1645 & 1.02 & 0.1648 \\
ATP4A & $\mathbf{2 . 2 3}$ & 0.7832 & 1.03 & 0.6392 & -1.03 & 0.2884 & -1.03 & 0.2967 \\
ATP4B & 1.45 & 0.3082 & 1.79 & 0.7798 & -1.21 & 0.2096 & 1.52 & 0.2148 \\
ATP5A1 & -1.08 & 0.9484 & -1.14 & 0.9730 & 1.06 & 0.7168 & 1.03 & 0.8362 \\
ATP5B & -1.05 & 0.9663 & -1.04 & 0.9969 & 1.00 & 0.9906 & 1.04 & 0.5569 \\
ATP5C1 & -1.05 & 0.9517 & -1.12 & 0.9898 & -1.08 & 0.8672 & -1.04 & 0.9454 \\
ATP5F1 & -1.23 & 0.1785 & 1.01 & 0.2102 & 1.05 & 0.1455 & 1.07 & 0.1458 \\
ATP5G1 & -1.02 & 0.8216 & 1.01 & 0.9565 & -1.05 & 0.1904 & 1.02 & 0.2007 \\
ATP5G2 & -1.15 & 0.8984 & 1.28 & 0.9757 & 1.28 & 0.2901 & 1.08 & 0.2901 \\
ATP5G3 & -1.04 & 0.2888 & -1.02 & 0.8484 & -1.09 & 0.1986 & -1.04 & 0.1983 \\
\hline
\end{tabular}


Table 1. Cont.

\begin{tabular}{|c|c|c|c|c|c|c|c|c|}
\hline Gene & $\begin{array}{l}\text { Inflamed vs. } \\
\text { Healthy }\end{array}$ & $p$ Value & $\begin{array}{l}\text { Light vs. } \\
\text { Inflamed }\end{array}$ & $p$ Value & $\begin{array}{l}\text { FLE-Gel vs. } \\
\text { Inflamed }\end{array}$ & $p$ Value & $\begin{array}{l}\text { FLE-Matrix } \\
\text { vs. Inflamed }\end{array}$ & $p$ Value \\
\hline ATP5H & -1.13 & 0.4352 & 1.05 & 0.7275 & 1.14 & 0.2591 & 1.08 & 0.2589 \\
\hline ATP5I & -1.08 & 0.9752 & 1.05 & 0.8689 & -1.05 & 0.8167 & 0.99 & 0.9525 \\
\hline ATP5J & 1.02 & 0.9968 & -1.02 & 0.9737 & -1.04 & 0.9612 & 0.99 & 0.9791 \\
\hline ATP5J2 & -1.23 & 0.9810 & -1.07 & 0.9603 & -1.01 & 0.2836 & 1.22 & 0.2834 \\
\hline ATP5L & 1.68 & 0.5228 & -1.36 & 0.8836 & -1.53 & 0.2096 & -1.27 & 0.2134 \\
\hline АTP5O & -1.24 & 0.8745 & 1.05 & 0.7809 & -1.03 & 0.8725 & -1.04 & 0.5821 \\
\hline ATP6V0A2 & 2.59 & 0.9270 & 1.03 & 0.3655 & -1.79 & 0.2746 & -1.09 & 0.1516 \\
\hline ATP6V0D2 & 1.91 & 0.6030 & 1.62 & 0.9800 & 1.36 & 0.2356 & 1.21 & 0.4197 \\
\hline ATP6V1C2 & 1.11 & 0.1784 & 1.12 & 0.6929 & -1.18 & 0.2758 & 1.20 & 0.2789 \\
\hline ATP6V1E2 & 1.02 & 0.6367 & 1.01 & 0.8071 & 1.17 & 0.1381 & 1.04 & 0.1352 \\
\hline ATP6V1G3 & -1.70 & 0.4560 & 1.12 & 0.8299 & -1.11 & 0.3228 & -1.05 & 0.3231 \\
\hline BCS1L & -1.46 & 0.5451 & -1.07 & 0.8080 & 1.16 & 0.4274 & 1.24 & 0.4942 \\
\hline COX4I1 & 1.08 & 0.6312 & -1.02 & 0.6719 & 1.03 & 0.7022 & 1.06 & 0.7194 \\
\hline COX4I2 & -1.85 & 0.9443 & 3.24 & 0.8211 & 1.40 & 0.1855 & 1.90 & 0.1854 \\
\hline COX5A & -1.15 & 0.9779 & -1.02 & 0.3636 & -1.05 & 0.1026 & 1.10 & 0.0556 \\
\hline COX5B & -1.08 & 0.8219 & 1.06 & 0.6803 & 1.01 & 0.6248 & 1.01 & 0.6164 \\
\hline COX6A1 & 1.05 & 0.9912 & -1.02 & 0.6452 & -1.05 & 0.9001 & -1.06 & 0.8699 \\
\hline COX6A2 & 1.29 & 0.7882 & 1.55 & 0.4056 & -1.03 & 0.5157 & -1.02 & 0.3269 \\
\hline COX6B1 & 1.09 & 0.8830 & 1.06 & 0.3851 & -1.12 & 0.3845 & -1.06 & 0.1568 \\
\hline COX6B2 & 1.54 & 0.6464 & 1.36 & 0.9643 & -1.06 & 0.2774 & 1.21 & 0.2775 \\
\hline COX6C & -2.23 & 0.2374 & 1.12 & 0.4507 & -1.11 & 0.2241 & 1.18 & 0.2246 \\
\hline COX7A2 & 1.31 & 0.8657 & 1.00 & 0.7080 & 1.00 & 0.2980 & 1.02 & 0.3576 \\
\hline$C O X 7 A 2 L$ & 1.14 & 0.9597 & 1.18 & 0.3701 & -1.02 & 0.2149 & 1.02 & 0.2810 \\
\hline СOX7B & -1.23 & 0.1399 & 1.03 & 0.9086 & -1.11 & 0.8089 & -1.07 & 0.8180 \\
\hline COX $8 A$ & -1.02 & 0.6213 & 1.04 & 0.7314 & -1.01 & 0.3849 & 1.03 & 0.4075 \\
\hline COX8C & 1.11 & 0.3632 & 3.60 & 0.3340 & -1.20 & 0.2921 & 1.67 & 0.2925 \\
\hline CYC1 & 1.03 & 0.9564 & 1.02 & 0.9543 & -1.01 & 0.8904 & 1.00 & 0.8846 \\
\hline LHPP & -1.12 & 0.7322 & 1.14 & 0.6447 & 1.10 & 0.9146 & 1.02 & 0.9953 \\
\hline NDUFA1 & 1.07 & 0.6609 & -1.06 & 0.7084 & 1.06 & 0.2131 & -1.16 & 0.2167 \\
\hline NDUFA10 & -1.06 & 0.9250 & 0.99 & 0.7389 & 1.01 & 0.6267 & 0.99 & 0.7675 \\
\hline NDUFA11 & -1.03 & 0.9466 & -1.02 & 0.9339 & 1.04 & 0.8978 & 1.09 & 0.9636 \\
\hline NDUFA2 & 1.07 & 0.9914 & 1.02 & 0.3434 & 1.11 & 0.1150 & 1.01 & 0.0749 \\
\hline NDUFA3 & -1.29 & 0.8155 & 1.05 & 0.9753 & -1.04 & 0.8924 & -1.03 & 0.8893 \\
\hline NDUFA4 & 1.09 & 0.5712 & 1.03 & 0.7243 & -1.04 & 0.0812 & -1.04 & 0.0819 \\
\hline NDUFA5 & -1.97 & 0.5816 & 1.28 & 0.9752 & -1.24 & 0.2903 & 1.15 & 0.3374 \\
\hline NDUFA6 & 1.03 & 0.1884 & -1.07 & 0.5686 & 1.18 & 0.1108 & 1.12 & 0.1032 \\
\hline NDUFA7 & -1.03 & 0.9399 & -1.03 & 0.9026 & 1.04 & 0.3395 & 1.00 & 0.2997 \\
\hline NDUFA8 & -1.23 & 0.8994 & -1.08 & 0.7818 & 1.23 & 0.8069 & 0.99 & 0.4317 \\
\hline NDUFAB1 & 1.08 & 0.5897 & 1.03 & 0.9813 & -1.03 & 0.7660 & -1.04 & 0.7574 \\
\hline NDUFB10 & -1.03 & 0.2064 & -1.03 & 0.6846 & 1.00 & 0.7707 & -1.02 & 0.7615 \\
\hline NDUFB2 & -1.15 & 0.9577 & 1.05 & 0.2898 & 1.05 & 0.1720 & -1.05 & 0.3253 \\
\hline NDUFB3 & -1.14 & 0.8127 & 1.03 & 0.8891 & 1.07 & 0.3763 & -1.03 & 0.3061 \\
\hline NDUFB4 & 1.24 & 0.3245 & -1.06 & 0.7046 & 1.09 & 0.0957 & 1.04 & 0.0861 \\
\hline NDUFB5 & -1.07 & 0.5122 & 1.04 & 0.1030 & 1.05 & 0.0993 & 1.03 & 0.0712 \\
\hline NDUFB6 & -1.08 & 0.6834 & 1.09 & 0.3958 & -1.07 & 0.4169 & -1.21 & 0.1946 \\
\hline NDUFB7 & -1.25 & 0.8837 & 1.03 & 0.9139 & 1.07 & 0.9146 & 1.11 & 0.9221 \\
\hline NDUFB8 & -1.29 & 0.9782 & -1.08 & 0.4260 & 1.04 & 0.0860 & 1.04 & 0.0867 \\
\hline NDUFB9 & -1.07 & 0.8154 & 1.04 & 0.6321 & -1.03 & 0.3974 & 1.00 & 0.3031 \\
\hline NDUFC1 & -1.13 & 0.6376 & 1.06 & 0.4091 & 1.10 & 0.1097 & -1.03 & 0.1077 \\
\hline NDUFC2 & 1.03 & 0.6633 & 1.03 & 0.5582 & 1.26 & 0.4491 & 1.21 & 0.6712 \\
\hline NDUFS1 & -1.05 & 0.8937 & 1.03 & 0.8077 & 1.06 & 0.2821 & 1.06 & 0.2782 \\
\hline NDUFS2 & -1.05 & 0.6046 & 1.08 & 0.8433 & 1.08 & 0.5569 & 1.05 & 0.5270 \\
\hline NDUFS3 & 1.14 & 0.7629 & -1.09 & 0.3173 & 1.05 & 0.1314 & 1.05 & 0.0777 \\
\hline NDUFS4 & -1.11 & 0.3228 & 1.05 & 0.6511 & -1.01 & 0.6848 & -1.04 & 0.5687 \\
\hline NDUFS5 & -1.13 & 0.9234 & 1.05 & 0.8181 & 1.01 & 0.3283 & -1.03 & 0.4957 \\
\hline NDUFS6 & -1.10 & 0.7346 & 1.09 & 0.9146 & 1.05 & 0.6572 & 1.11 & 0.6882 \\
\hline NDUFS7 & -1.29 & 0.7148 & -1.32 & 0.7148 & 1.05 & 0.3395 & 1.02 & 0.2788 \\
\hline NDUFS8 & -1.23 & 0.8670 & 1.03 & 0.9886 & 1.18 & 0.9448 & 1.23 & 0.9275 \\
\hline NDUFV1 & -1.21 & 0.8922 & 0.99 & 0.6639 & 1.04 & 0.1205 & 1.06 & 0.1277 \\
\hline NDUFV2 & -1.21 & 0.8671 & 1.08 & 0.5305 & 1.06 & 0.1460 & -1.02 & 0.2872 \\
\hline NDUFV3 & 1.13 & 0.7272 & -1.10 & 0.3081 & 1.26 & 0.1167 & 1.24 & 0.1145 \\
\hline OXA1L & -1.13 & 0.5362 & 1.14 & 0.5885 & 1.27 & 0.1040 & 1.22 & 0.1026 \\
\hline
\end{tabular}


Table 1. Cont.

\begin{tabular}{ccccccccc}
\hline Gene & $\begin{array}{c}\text { Inflamed vs. } \\
\text { Healthy }\end{array}$ & $p$ Value & $\begin{array}{c}\text { Light vs. } \\
\text { Inflamed }\end{array}$ & $p$ Value & $\begin{array}{c}\text { FLE-Gel vs. } \\
\text { Inflamed }\end{array}$ & $p$ Value & $\begin{array}{c}\text { FLE-Matrix } \\
\text { vs. Inflamed }\end{array}$ & $p$ Value \\
\hline PPA1 & -1.71 & 0.2951 & -1.03 & 0.5926 & 1.04 & 0.8757 & -1.02 & 0.8405 \\
PPA2 & -1.19 & 0.5189 & 1.04 & 0.4868 & -1.02 & 0.2007 & -1.04 & 0.2018 \\
SDHA & -1.42 & 0.3281 & 1.09 & 0.5285 & 1.05 & 0.1407 & 1.02 & 0.1423 \\
SDHB & 1.04 & 0.5917 & 1.01 & 0.8479 & 1.04 & 0.6252 & 1.03 \\
SDHC & -1.19 & 0.7466 & 1.22 & 0.6577 & -1.05 & 0.1798 & -1.02 \\
SDHD & -1.22 & 0.8702 & -1.23 & 0.9003 & 1.00 & 0.5197 & -1.07 \\
UQCR11 & 1.26 & 0.4176 & -1.16 & 0.8663 & -1.04 & 0.1969 & -1.06 \\
UQCRC1 & -1.03 & 0.9857 & -1.13 & 0.8575 & -1.02 & 0.9116 & -1.04 \\
UQCRC2 & -1.29 & 0.8090 & -1.21 & 0.1460 & 1.02 & 0.1357 & 0.1799 \\
UQCRFS1 & -1.03 & 0.9645 & -1.17 & 0.8910 & -1.03 & 0.9580 & -1.04 \\
UQCRH & -1.03 & 0.9254 & -1.07 & 0.9792 & -1.04 & 0.9931 & -1.04 \\
UQCRQ & 1.00 & 0.5753 & -1.11 & 0.7945 & 1.06 & 0.8875 & 0.1405 \\
\hline
\end{tabular}

ATP12A: ATPase, $\mathrm{H}+/ \mathrm{K}+$ transporting, nongastric, alpha polypeptide; ATP4A: ATPase, $\mathrm{H}+\mathrm{K}+$ exchanging, alpha polypeptide; ATP4B: ATPase, $\mathrm{H}+/ \mathrm{K}+$ exchanging, beta polypeptide; ATP5A1: ATP synthase, $\mathrm{H}+$ transporting, mitochondrial F1 complex, alpha subunit 1, cardiac muscle; ATP5B: ATP synthase, $\mathrm{H}+$ transporting, mitochondrial F1 complex, beta polypeptide; ATP5C1: ATP synthase, $\mathrm{H}+$ transporting, mitochondrial F1 complex, gamma polypeptide 1; ATP5F1: ATP synthase, $\mathrm{H}+$ transporting, mitochondrial Fo complex, subunit B1; ATP5G1: ATP synthase, $\mathrm{H}+$ transporting, mitochondrial Fo complex, subunit C1; ATP5G2: ATP synthase, H+ transporting, mitochondrial Fo complex, subunit C2; ATP5G3: ATP synthase, H+ transporting, mitochondrial Fo complex, subunit C3; ATP5H: ATP synthase, $\mathrm{H}+$ transporting, mitochondrial Fo complex, subunit d; ATP5I: ATP synthase, $\mathrm{H}+$ transporting, mitochondrial Fo complex, subunit E; ATP5J: ATP synthase, $\mathrm{H}+$ transporting, mitochondrial Fo complex, subunit F6; ATP5J2: ATP synthase, H+ transporting, mitochondrial Fo complex, subunit F2; ATP5L: ATP synthase, $\mathrm{H}+$ transporting, mitochondrial Fo complex, subunit G; ATP5O: ATP synthase, $\mathrm{H}+$ transporting, mitochondrial F1 complex, O subunit; ATP6V0A2: ATPase, H+ transporting, lysosomal V0 subunit a2; ATP6V0D2: ATPase, $\mathrm{H}+$ transporting, lysosomal 38 kDa, V0 subunit d2; ATP6 V1C2: ATPase, $\mathrm{H}+$ transporting, lysosomal 42 $\mathrm{kDa}$, V1 subunit C2; ATP6V1E2: ATPase, $\mathrm{H}+$ transporting, lysosomal $31 \mathrm{kDa}, \mathrm{V} 1$ subunit E2; ATP6V1G3:ATPase, $\mathrm{H}+$ transporting, lysosomal $13 \mathrm{kDa}$, V1 subunit G3; BCS1L: BCS1-like (S. cerevisiae); COX4I1: Cytochrome c oxidase subunit IV isoform 1; COX4I2: Cytochrome c oxidase subunit IV isoform 2; COX5A: Cytochrome c oxidase subunit Va; COX5B: Cytochrome c oxidase subunit Vb; COX6A1: Cytochrome c oxidase subunit VIa polypeptide 1; COX6A2: Cytochrome c oxidase subunit VIa polypeptide 2; COX6B1: Cytochrome c oxidase subunit Vib polypeptide 1; COX6B2: Cytochrome c oxidase subunit VIb polypeptide 2; COX6C: Cytochrome c oxidase subunit Vic; COX7A2: Cytochrome c oxidase subunit VIIa polypeptide 2; COX7A2L: Cytochrome c oxidase subunit VIIa polypeptide 2 like; COX7B: Cytochrome c oxidase subunit VIIb; COX8A: Cytochrome c oxidase subunit VIIIA; COX8C: Cytochrome c oxidase subunit VIIIC; CYC1: Cytochrome c-1; LHPP: Phospholysine phosphohistidine inorganic pyrophosphate phosphatase; NDUFA1: NADH dehydrogenase 1 alpha subcomplex, 1, 7.5 kDa; NDUFA10: NADH dehydrogenase 1 alpha subcomplex, 10, $42 \mathrm{kDa}$; NDUFA11: NADH dehydrogenase 1 alpha subcomplex, 11, $14.7 \mathrm{kDa}$; NDUFA2: NADH dehydrogenase 1 alpha subcomplex, 2, $8 \mathrm{kDa}$; NDUFA3: NADH dehydrogenase 1 alpha subcomplex, 3, $9 \mathrm{kDa}$; NDUFA4: NADH dehydrogenase 1 alpha subcomplex, 4, $9 \mathrm{kDa}$; NDUFA5: NADH dehydrogenase 1 alpha subcomplex, 5, 13 kDa; NDUFA6: NADH dehydrogenase 1 alpha subcomplex, 6, 14 kDa; NDUFA7: NADH dehydrogenase 1 alpha subcomplex, 7, $14.5 \mathrm{kDa}$; NDUFA8: NADH dehydrogenase 1 alpha subcomplex, 8, 19 kDa; NDUFAB1: NADH dehydrogenase 1, alpha/beta subcomplex, 1, 8 kDa; NDUFB10: NADH dehydrogenase 1 beta subcomplex, 10, $22 \mathrm{kDa}$; NDUFB2: NADH dehydrogenase 1 beta subcomplex, 2, $8 \mathrm{kDa}$; NDUFB3: NADH dehydrogenase 1 beta subcomplex, 3, $12 \mathrm{kDa}$; NDUFB4: NADH dehydrogenase 1 beta subcomplex, 4, $15 \mathrm{kDa}$; NDUFB5: NADH dehydrogenase 1 beta subcomplex, 5, 16 kDa; NDUFB6: NADH dehydrogenase 1 beta subcomplex, 6, $17 \mathrm{kDa}$; NDUFB7: NADH dehydrogenase 1 beta subcomplex, 7, $18 \mathrm{kDa}$; NDUFB8: NADH dehydrogenase 1 beta subcomplex, 8, $19 \mathrm{kDa}$; NDUFB9: NADH dehydrogenase 1 beta subcomplex, 9, $22 \mathrm{kDa}$; NDUFC1: NADH dehydrogenase 1, subcomplex unknown, 1, $6 \mathrm{kDa}$; NDUFC2: NADH dehydrogenase 1, subcomplex unknown, 2, $14.5 \mathrm{kDa}$; NDUFS1: NADH dehydrogenase Fe-S protein 1, $75 \mathrm{kDa}$; NDUFS2: NADH dehydrogenase Fe-S protein 2, $49 \mathrm{kDa}$; NDUFS3: NADH dehydrogenase Fe-S protein 3, $30 \mathrm{kDa}$; NDUFS4: NADH dehydrogenase Fe-S protein 4, $18 \mathrm{kDa}$; NDUFS5: NADH dehydrogenase Fe-S protein 5, $15 \mathrm{kDa}$; NDUFS6: NADH dehydrogenase Fe-S protein 6, $13 \mathrm{kDa}$; NDUFS7: NADH dehydrogenase Fe-S protein 7, $20 \mathrm{kDa}$; NDUFS8: NADH dehydrogenase Fe-S protein 8, $23 \mathrm{kDa}$; NDUFV1: NADH dehydrogenase flavoprotein 1, $51 \mathrm{kDa}$; NDUFV2: NADH dehydrogenase flavoprotein 2, 24 kDa; NDUFV3: NADH dehydrogenase flavoprotein 3, $10 \mathrm{kDa}$; OXA1 L: Oxidase (cytochrome c) assembly 1-like; PPA1: Pyrophosphatase 1; PPA2: Pyrophosphatase 2; SDHA: Succinate dehydrogenase complex, subunit A, flavoprotein (Fp); SDHB: Succinate dehydrogenase complex, subunit B, iron sulfur (Ip); SDHC: Succinate dehydrogenase complex, subunit C, integral membrane protein, $15 \mathrm{kDa}$; SDHD: Succinate dehydrogenase complex, subunit D, integral membrane protein; UQCR11: Ubiquinol-cy tochrome c reductase, complex III subunit XI; UQCRC1: Ubiquinol-cytochrome c reductase core protein I; UQCRC2: Ubiquinol-cytochrome c reductase core protein II; UQCRFS1: Ubiquinol-cytochrome c reductase, Rieske iron-sulfur polypeptide 1; UQCRH: Ubiquinol-cytochrome c reductase hinge protein; UQCRQ: Ubiquinol-cytochrome c reductase, complex III subunit VII, $9.5 \mathrm{kDa}$.

Of note, compared with Healthy HDFs, Inflamed HDFs had upregulated ATP4A and ATP6V0A2 genes and downregulated $\mathrm{COX6C}$ gene. These genes encode enzymes belonging to ATP synthase and $\mathrm{CCO}$, respectively, which are fundamental for energy production and oxidative phosphorylation. 
The remaining 81 investigated genes were expressed at similar levels in both conditions. Compared with Inflamed cells, no treatment altered the expression of these genes.

Compared with Inflamed HDFs, cells treated with Light overexpressed two genes belonging to CCO: COX4I2 and COX8C. These genes remained unchanged in both cell group treated with FLE.

Genes belonging to complex I (NADH-Coenzyme Q Reductase), complex II (Succinate-Coenzyme $\mathrm{Q}$ Reductase), and complex III (Coenzyme Q-Cytochrome c Reductase) maintained the same expression profile in all the conditions investigated.

Table 2 reports the expression profiles of 84 genes related to biogenesis and function of mitochondria, including regulators and mediators of mitochondrial molecular transport of metabolites required for the electron transport chain and oxidative phosphorylation.

Table 2. Human Mitochondria PCR array.

\begin{tabular}{|c|c|c|c|c|c|c|c|c|}
\hline Gene & $\begin{array}{c}\text { Inflamed } \\
\text { vs. } \\
\text { Healthy }\end{array}$ & $p$ Value & $\begin{array}{l}\text { Light vs } \\
\text { Inflamed }\end{array}$ & $p$ Value & $\begin{array}{l}\text { FLE-Gel } \\
\text { vs. } \\
\text { Inflamed } \\
\end{array}$ & $p$ Value & $\begin{array}{l}\text { FLE-Matrix } \\
\text { vs. } \\
\text { Inflamed }\end{array}$ & $p$ Value \\
\hline AIFM2 & 1.17 & 0.9186 & 1.05 & 0.9694 & 1.15 & 0.8808 & 1.05 & 0.3564 \\
\hline AIP & -1.12 & 0.9070 & 1.16 & 0.8602 & 1.21 & 0.9389 & 1.13 & 0.8818 \\
\hline$B A K 1$ & 2.18 & 0.4666 & -1.09 & 0.8772 & 1.02 & 0.7111 & -1.05 & 0.1289 \\
\hline$B B C 3$ & -2.91 & 0.9816 & 1.31 & 0.9722 & 1.08 & 0.9145 & 1.04 & 0.6868 \\
\hline BCL2 & 1.93 & 0.4330 & 1.13 & 0.4945 & 1.14 & 0.4947 & 1.16 & 0.5045 \\
\hline$B C L 2 L 1$ & -1.37 & 0.9929 & 1.07 & 0.4951 & -1.07 & 0.3020 & -1.07 & 0.3250 \\
\hline BID & 1.51 & 0.6882 & 1.01 & 0.8719 & 1.06 & 0.9178 & -1.35 & 0.2987 \\
\hline BNIP3 & -1.36 & 0.8372 & 1.06 & 0.9668 & -1.23 & 0.9024 & -1.16 & 0.9280 \\
\hline$C D K N 2 A$ & -2.99 & 0.7208 & -1.03 & 0.5949 & 1.13 & 0.3788 & -1.23 & 0.3111 \\
\hline COX10 & -1.03 & 0.6641 & 1.01 & 0.4646 & -1.10 & 0.5937 & -1.08 & 0.3185 \\
\hline COX18 & -1.36 & 0.7253 & 1.32 & 0.6170 & 1.30 & 0.4723 & 1.09 & 0.3871 \\
\hline СРТ1В & -1.33 & 0.8146 & 0.99 & 0.9582 & 1.82 & 0.9127 & 2.04 & 0.4682 \\
\hline СРТ2 & 1.11 & 0.6167 & 1.11 & 0.5475 & 1.04 & 0.4144 & -1.03 & 0.3732 \\
\hline DNM1L & 1.04 & 0.6504 & 1.02 & 0.4551 & -1.02 & 0.3857 & 1.01 & 0.3536 \\
\hline FIS1 & -1.29 & 0.7077 & 1.12 & 0.4843 & 1.15 & 0.4490 & 1.07 & 0.3857 \\
\hline GRPEL1 & 1.40 & 0.9575 & -1.15 & 0.8785 & -1.12 & 0.3880 & -1.01 & 0.8202 \\
\hline HSP90AA1 & -1.19 & 0.8602 & 1.06 & 0.5892 & 1.13 & 0.1296 & 1.27 & 0.4059 \\
\hline HSPD1 & -1.17 & 0.8993 & -1.01 & 0.9746 & 1.00 & 0.9350 & 1.07 & 0.8728 \\
\hline$I M M P 1 L$ & -2.51 & 0.9134 & -1.02 & 0.9908 & 1.06 & 0.9928 & 1.10 & 0.9780 \\
\hline$I M M P 2 L$ & -1.31 & 0.5724 & 1.08 & 0.9491 & 1.08 & 0.9692 & 1.06 & 0.4700 \\
\hline$L R P P R C$ & -1.20 & 0.9990 & 1.19 & 0.7798 & 1.07 & 0.6555 & 1.10 & 0.6068 \\
\hline MFN1 & -1.07 & 0.7624 & 1.05 & 0.9604 & 1.11 & 0.8119 & 1.10 & 0.5266 \\
\hline MFN2 & -1.09 & 0.9807 & -1.11 & 0.7044 & -1.15 & 0.5278 & -1.06 & 0.5023 \\
\hline MIPEP & -1.84 & 0.9939 & -1.03 & 0.8895 & 1.23 & 0.9541 & 1.26 & 0.4258 \\
\hline$M P V 17$ & -1.56 & 0.9050 & 1.05 & 0.9991 & 1.06 & 0.9772 & 1.04 & 0.5992 \\
\hline MSTO1 & 1.04 & 0.9795 & 1.10 & 0.9732 & 1.26 & 0.6936 & 1.17 & 0.3500 \\
\hline MTX2 & 1.01 & 0.4066 & 1.07 & 0.4383 & 1.33 & 0.2515 & 1.28 & 0.1868 \\
\hline NEFL & 16.93 & 0.6088 & -1.36 & 0.9775 & -1.65 & 0.4401 & -1.51 & 0.3560 \\
\hline OPA1 & -1.20 & 0.5516 & 1.15 & 0.9987 & 1.10 & 0.6269 & 1.15 & 0.9292 \\
\hline PMAIP1 & -1.06 & 0.8934 & -1.01 & 0.9052 & -1.24 & 0.9505 & -1.03 & 0.9594 \\
\hline RHOT1 & -1.42 & 0.8552 & 1.13 & 0.6221 & 1.20 & 0.4302 & 1.17 & 0.3495 \\
\hline RHOT2 & 1.07 & 0.7768 & 1.03 & 0.5066 & 1.00 & 0.5196 & 1.00 & 0.9821 \\
\hline SFN & -2.38 & 0.4837 & 1.66 & 0.8181 & 1.90 & 0.4771 & 1.75 & 0.4248 \\
\hline SH3GLB1 & -1.22 & 0.9383 & 1.28 & 0.9551 & 1.52 & 0.8607 & 1.52 & 0.3748 \\
\hline SLC25A1 & -2.07 & 0.8876 & 1.13 & 0.8759 & 1.05 & 0.7808 & 1.04 & 0.7874 \\
\hline SLC25A10 & 1.38 & 0.6742 & -1.62 & 0.9591 & -1.85 & 0.9908 & -1.60 & 0.9927 \\
\hline SLC25A12 & -1.26 & 0.8391 & -1.36 & 0.9808 & -1.22 & 0.9588 & -1.12 & 0.3523 \\
\hline SLC25A13 & 1.51 & 0.8949 & 1.11 & 0.9549 & -1.05 & 0.9313 & 1.09 & 0.3061 \\
\hline SLC25A14 & 1.13 & 0.5778 & 1.06 & 0.9728 & 1.04 & 0.9826 & 1.03 & 0.2791 \\
\hline SLC25A15 & 1.20 & 0.6720 & -1.02 & 0.5093 & 1.18 & 0.4814 & 1.21 & 0.3807 \\
\hline SLC25A16 & -1.12 & 0.9002 & 1.13 & 0.8996 & 1.03 & 0.8461 & 1.16 & 0.6215 \\
\hline
\end{tabular}


Table 2. Cont.

\begin{tabular}{|c|c|c|c|c|c|c|c|c|}
\hline Gene & $\begin{array}{c}\text { Inflamed } \\
\text { vs. } \\
\text { Healthy }\end{array}$ & $p$ Value & $\begin{array}{l}\text { Light vs } \\
\text { Inflamed }\end{array}$ & $p$ Value & $\begin{array}{l}\text { FLE-Gel } \\
\text { vs. } \\
\text { Inflamed }\end{array}$ & $p$ Value & $\begin{array}{l}\text { FLE-Matrix } \\
\text { vs. } \\
\text { Inflamed }\end{array}$ & $p$ Value \\
\hline SLC25A17 & 1.26 & 0.8423 & -1.05 & 0.9918 & -1.10 & 0.8879 & -1.06 & 0.5068 \\
\hline SLC25A19 & 1.44 & 0.8739 & -1.08 & 0.9144 & -1.07 & 0.8900 & -1.06 & 0.9309 \\
\hline SLC25A2 & 1.14 & 0.6760 & 1.17 & 0.4763 & 1.15 & 0.4239 & 1.48 & 0.3471 \\
\hline SLC25A20 & -1.19 & 0.8596 & -1.14 & 0.7744 & 1.05 & 0.8105 & 1.05 & 0.5398 \\
\hline SLC25A21 & -1.42 & 0.8432 & 0.99 & 0.5065 & 1.30 & 0.5826 & 1.43 & 0.3696 \\
\hline SLC25A22 & 1.21 & 0.7463 & 1.07 & 0.6007 & 1.11 & 0.6098 & 1.17 & 0.3715 \\
\hline SLC25A23 & -1.43 & 0.7167 & -1.09 & 0.4906 & -1.52 & 0.4234 & -1.27 & 0.3587 \\
\hline SLC25A24 & -1.13 & 0.8747 & 1.05 & 0.8545 & 1.02 & 0.9513 & 1.03 & 0.3730 \\
\hline SLC25A25 & 2.30 & 0.9570 & -1.06 & 0.9910 & -1.11 & 0.8293 & -1.09 & 0.6173 \\
\hline SLC25A27 & -1.27 & 0.3919 & -2.25 & 0.9605 & -1.10 & 0.4895 & -1.55 & 0.7273 \\
\hline SLC25A3 & -1.20 & 0.3646 & 1.02 & 0.7269 & 1.03 & 0.4790 & 1.01 & 0.1843 \\
\hline SLC25A30 & -1.36 & 0.9004 & 1.12 & 0.9875 & 1.08 & 0.9820 & 1.10 & 0.9916 \\
\hline SLC25A31 & -1.12 & 0.8581 & 1.28 & 0.9795 & 2.47 & 0.9464 & -1.06 & 0.4897 \\
\hline SLC25A37 & 1.92 & 0.6504 & 1.07 & 0.9261 & 1.21 & 0.8353 & 1.24 & 0.8423 \\
\hline SLC25A4 & -1.86 & 0.6059 & 1.06 & 0.8584 & -1.03 & 0.6999 & 1.02 & 0.8379 \\
\hline SLC25A5 & 1.00 & 0.9160 & -1.09 & 0.5923 & 1.01 & 0.5904 & 1.05 & 0.3863 \\
\hline SOD1 & -1.14 & 0.6756 & 1.09 & 0.4430 & 1.08 & 0.4504 & 1.14 & 0.3994 \\
\hline SOD2 & 17.28 & 0.8853 & 1.28 & 0.7959 & 1.19 & 0.7786 & 1.13 & 0.7637 \\
\hline STARD3 & -1.04 & 0.3845 & 1.00 & 0.8834 & -1.02 & 0.9115 & 1.01 & 0.9488 \\
\hline $\mathrm{TAZ}$ & -1.26 & 0.8577 & 1.02 & 0.3116 & 1.27 & 0.2549 & 1.22 & 0.9748 \\
\hline TIMM10 & 1.22 & 0.8033 & -1.08 & 0.5207 & -1.09 & 0.4573 & 1.03 & 0.3611 \\
\hline TIMM10B & 1.14 & 0.7175 & 1.06 & 0.9674 & 1.01 & 0.6375 & -1.01 & 0.7582 \\
\hline TIMM17A & 1.22 & 0.7552 & -1.08 & 0.7767 & 1.05 & 0.7586 & 1.01 & 0.7073 \\
\hline TIMM17B & -1.33 & 0.7433 & 1.29 & 0.5761 & 1.15 & 0.5304 & 1.23 & 0.4434 \\
\hline TIMM22 & -1.06 & 0.7358 & -1.08 & 0.4982 & 1.00 & 0.4019 & 1.17 & 0.3696 \\
\hline TIMM23 & 1.07 & 0.9080 & -1.05 & 0.9481 & 1.00 & 0.9475 & -1.01 & 0.7871 \\
\hline TIMM44 & 1.13 & 0.7086 & -1.06 & 0.5628 & -1.14 & 0.7906 & -1.13 & 0.3392 \\
\hline TIMM50 & -1.06 & 0.9866 & 1.09 & 0.8853 & 1.00 & 0.9968 & 1.09 & 0.8634 \\
\hline TIMM8A & 1.28 & 0.7994 & -1.11 & 0.1517 & -1.05 & 0.2349 & -1.01 & 0.2730 \\
\hline TIMM8B & -1.11 & 0.9864 & -1.06 & 0.9095 & -1.09 & 0.8707 & 1.02 & 0.9847 \\
\hline TIMM9 & 1.37 & 0.6803 & -1.14 & 0.6204 & -1.29 & 0.4490 & -1.33 & 0.3385 \\
\hline TOMM20 & 1.07 & 0.9647 & 1.00 & 0.9962 & -1.05 & 0.9785 & -1.03 & 0.9860 \\
\hline TOMM22 & -1.03 & 0.9395 & -1.03 & 0.9564 & -1.05 & 0.9226 & 1.00 & 0.8254 \\
\hline TOMM34 & 1.16 & 0.9339 & 1.04 & 0.9646 & 0.99 & 0.9878 & 1.08 & 0.9486 \\
\hline TOMM40 & 1.29 & 0.5140 & -1.16 & 0.6759 & -1.09 & 0.8690 & 0.99 & 0.7455 \\
\hline TOMM40L & -1.01 & 0.9861 & 1.13 & 0.8819 & 1.05 & 0.6619 & 1.15 & 0.3629 \\
\hline TOMM70A & -1.16 & 0.9512 & 1.07 & 0.9631 & 1.19 & 0.8725 & 1.17 & 0.3903 \\
\hline ТР53 & -2.04 & 0.7434 & 1.44 & 0.8865 & 1.27 & 0.5986 & -1.05 & 0.3399 \\
\hline TSPO & -1.30 & 0.8269 & 1.10 & 0.5675 & 1.02 & 0.4945 & 1.00 & 0.3463 \\
\hline UCP1 & -1.52 & 0.8095 & 3.89 & 0.8001 & -1.09 & 0.6418 & 2.70 & 0.3743 \\
\hline UCP2 & -1.52 & 0.9517 & 1.10 & 0.9361 & 1.08 & 0.8185 & 1.09 & 0.3625 \\
\hline UCP3 & 1.59 & 0.7862 & 1.64 & 0.6184 & -1.31 & 0.5903 & 1.48 & 0.4868 \\
\hline UXT & 1.07 & 0.9794 & 1.09 & 0.6893 & 1.05 & 0.8536 & 1.02 & 0.4300 \\
\hline
\end{tabular}

AIFM2: Apoptosis-inducing factor, mitochondrion-associated, 2; AIP: Aryl hydrocarbon receptor interacting protein; BAK1: BCL2-antagonist/killer 1; BBC3: BCL2 binding component 3; BCL2: B-cell CLL/lymphoma 2; BCL2L1: BCL2-like 1; BID: BH3 interacting domain death agonist; BNIP3: BCL2/adenovirus E1B 19 kDa interacting protein 3; CDKN2A: Cyclin-dependent kinase inhibitor 2A; COX10: COX10 homolog, cytochrome c oxidase assembly protein; COX18: COX18 cytochrome c oxidase assembly homolog; CPT1B: Carnitine palmitoyltransferase 1B; CPT2: Carnitine palmitoyltransferase 2; DNM1L: Dynamin 1-like; FIS1: Fission 1; GRPEL1: GrpE-like 1, mitochondrial; HSP90AA1: Heat shock protein $90 \mathrm{kDa}$ alpha, class A member 1; HSPD1: Heat shock $60 \mathrm{kDa}$ protein 1; IMMP1L: IMP1 inner mitochondrial membrane peptidase-like; IMMP2L: IMP2 inner mitochondrial membrane peptidase-like; LRPPRC: Leucine-rich PPR-motif containing; MFN1: Mitofusin 1; MFN2: Mitofusin 2; MIPEP: Mitochondrial intermediate peptidase; MPV17: MpV17 mitochondrial inner membrane protein; MSTO1: Misato homolog 1; MTX2: Metaxin 2; NEFL: Neurofilament, light polypeptide; OPA1: Optic atrophy 1; PMAIP1: Phorbol-12-myristate-13-acetate-induced protein 1; RHOT1: Ras homolog gene family, member T1; RHOT2: Ras homolog gene family, member T2; SFN: Stratifin; SH3GLB1: SH3-domain GRB2-like endophilin B1; SLC25A1: Solute carrier family 25 (mitochondrial carrier; citrate transporter), member 1; SLC25A10: Solute carrier family 25, member 10; SLC25A12: Solute carrier family 25, member 12; SLC25A13: Solute carrier family 25, member 13; SLC25A14: Solute carrier family 25, member 14; SLC25A15: Solute carrier family 25, member 15; SLC25A16: Solute carrier family 25, member 16; SLC25A17: Solute 
carrier family 25, member 17; SLC25A19: Solute carrier family 25, member 19; SLC25A2: Solute carrier family 25, member 2; SLC25A20: Solute carrier family 25, member 20; SLC25A21: Solute carrier family 25, member 21; SLC25A22: Solute carrier family 25, member 22; SLC25A23: Solute carrier family 25, member 23; SLC25A24: Solute carrier family 25, member 24; SLC25A25: Solute carrier family 25, member 25; SLC25A27: Solute carrier family 25, member 27; SLC25A3: Solute carrier family 25, member 3; SLC25A30: Solute carrier family 25, member 30; SLC25A31: Solute carrier family 25, member 31; SLC25A37: Solute carrier family 25, member 37; SLC25A4: Solute carrier family 25, member 4; SLC25A5: Solute carrier family 25, member 5; SOD1: Superoxide dismutase 1; SOD2: Superoxide dismutase 2; STARD3: StAR-related lipid transfer (START) domain containing 3; TAZ: Tafazzin; TIMM10: Translocase of inner mitochondrial membrane 10 homolog; TIMM10B: Fracture callus 1 homolog; TIMM17A: Translocase of inner mitochondrial membrane 17 homolog A; TIMM17B: Translocase of inner mitochondrial membrane 17 homolog B; TIMM22: Translocase of inner mitochondrial membrane 22 homolog; TIMM23: Translocase of inner mitochondrial membrane 23 homolog; TIMM44:Translocase of inner mitochondrial membrane 44 homolog; TIMM50: Translocase of inner mitochondrial membrane 50 homolog; TIMM8A: Translocase of inner mitochondrial membrane 8 homolog A; TIMM8B: Translocase of inner mitochondrial membrane 8 homolog B; TIMM9: Translocase of inner mitochondrial membrane 9 homolog; TOMM20: Translocase of outer mitochondrial membrane 20 homolog; TOMM22: Translocase of outer mitochondrial membrane 22 homolog; TOMM34: Translocase of outer mitochondrial membrane 34; TOMM40: Translocase of outer mitochondrial membrane 40 homolog; TOMM40L: Translocase of outer mitochondrial membrane 40 homolog-like; TOMM70A: Translocase of outer mitochondrial membrane 70 homolog A; TP53: Tumor protein p53; TSPO: Translocator protein (18 kDa); UCP1: Uncoupling protein 1; UCP2: Uncoupling protein 2; UCP3: Uncoupling protein 3; UXT: Ubiquitously-expressed transcript.

Compared to Healthy cells, Inflamed HDFs showed substantial upregulation of superoxide dismutase 2 (SOD2) and neurofilament (NEFL) genes. SOD2 is an antioxidant enzyme that protects cells from oxidative damage, whereas NEFL influences the dynamics of mitochondria. Although to a lesser extent, an upregulation of BCL2-antagonist/killer 1 (BAK1) and solute carrier family 25, member 25 (SLC25A25) and a downregulation of BCL2 binding component 3 (BBC3), Cyclin-dependent kinase inhibitor 2A (CDKN2A), IMP1 inner mitochondrial membrane peptidase-like (IMMP1L), stratifin (SFN), solute carrier family 25 (mitochondrial carrier; citrate transporter), member 1 (SLC25A1), and tumor protein p53 (PT53) were also observed. The BAK1 gene encodes a pro-apoptotic protein. SLC25A25 and SLC25A1 genes encode proteins belonging to the family of calcium-binding mitochondrial carriers in the inner membranes of the mitochondria. Their functions are to transport proteins, metabolites, nucleotides and cofactors through the mitochondrial membrane and thereby connect and/or regulate cytoplasm and matrix functions [46]. The SFN gene encodes the stratifin protein, a cell cycle checkpoint protein that binds translation and initiation factors and functions as a regulator of mitotic translation. It also regulates signal transduction pathways and cellular trafficking [47]. Cyclin-dependent kinase inhibitor 2A (CDKN2A) is an inhibitor of CDK4 kinase and arrests the cell cycle in G1 phase [48]. The $B B C 3$ gene encodes the pro-apoptotic protein PUMA that is regulated by the protein tumor suppressor p53 [49]. The PT53 gene encodes p53 protein that exhibits diverse and global functions, including cell cycle arrest, senescence, and apoptosis. Through these pathways, p53 facilitates the repair and survival of damaged cells or eliminates severely injured cells from the replicative pool to protect the organism. One of the most dramatic responses to p53 activation is the induction of apoptosis [50]. No treatment altered any of the above-mentioned genes compared with Inflamed HDFs.

Of interest, an upregulation of genes involved in the production of ATP and reduction of ROS was observed. Compared to Inflamed cells, Light and FLE-Matrix treated HDFs showed an increase in UCP1 gene expression. This gene encodes uncoupling protein 1, a member of the family of mitochondrial anion carrier proteins. The uncoupling proteins (UCPs) separate oxidative phosphorylation from ATP synthesis with energy dissipated as heat, also referred to as mitochondrial proton leak. UCPs facilitate the transfer of anions from the inner to the outer mitochondrial membrane and the return transfer of protons from the outer to the inner mitochondrial membrane reducing the mitochondrial membrane potential. A minor decline in the mitochondrial membrane potential leads to a significant decrease in harmful levels of ROS production. UCPs proteins decrease mitochondrial membrane potential to a level still allowing both production of required amounts of ATP, and of lower ROS levels that would be relatively harmless to the cells [51]. Light treated cells also had a reduction in the expression of SLC25A27, alias UCP4. Moreover, compared to Inflamed HDFs, FLE-Matrix treated cells had an upregulation of $C P T 1 B$ gene. Carnitine palmitoyltransferase-1 (CPT1) is located in the inner aspect of the outer mitochondrial membrane and transports long-chain fatty acids into mitochondria for 
$\beta$-oxidation. The acetyl-coenzyme A (acetyl- CoA) produced by oxidative degradation of fatty acids enters the citric acid cycle for oxidation to carbon dioxide and water by the electron transport chain to yield ATP [52]. Compared to Inflamed cells, the FLE-Gel treated HDFs showed an increase in SLC25A31 gene expression. The protein encoded by this gene is a member of the ADP/ATP carrier family of proteins that exchange cytosolic ADP for matrix ATP in the mitochondria [53].

\section{Discussion}

In recent years, FLE has been demonstrated to be a safe and effective non-pharmacological intervention for the treatment of acute and chronic wounds [23,37,39] and other skin pathologies $[41,42]$, however, the exact mechanism through which FLE realizes an acceleration in the wound healing process is not yet clear.

The principle behind FLE is the absorption of photons by endogenous chromophores inside the treated tissues, resulting in various biological effects [36]. It is widely known that mitochondria are an initial site of light action in cells, and the central molecule for this is $\mathrm{CCO}$, the terminal enzyme of the mitochondrial respiratory chain. CCO transfers one electron (from each of four cytochrome $c$ molecules), to a single oxygen molecule, producing two molecules of water. At the same time the four protons required are translocated across the mitochondrial membrane, producing a proton gradient that ATP synthase uses to synthesize ATP [54]. CCO has two heme centers and two copper centers. Each of these metal centers can exist in an oxidized or a reduced state, and each are photo-acceptors with different absorption spectra in the red and near-infrared region (up to $950 \mathrm{~nm}$ ). The absorption of photons by CCO leads to an increase in enzymatic activity, an increase in oxygen consumption, and an increase in ATP production thanks to the photodissociation of inhibitory nitric oxide (NO) [55]. Since $\mathrm{NO}$ is non-covalently bound to the heme and copper centers and competitively blocks oxygen at a ratio of 1:10, a relatively low energy photon can move the NO, allowing a lot of respiration to take place [56].

This theory describes the structural changes that CCO undergoes after exposure to photons. Conversely, the present work aims to investigate the mitochondrial morphology and the expression profile of genes related to the respiratory chain, and the mitochondrial dynamics and functions in inflamed cells after the exposure to photons as either direct light (PBM) or FLE. For this purpose, in vitro cultures of HDFs were stimulated overnight with a cocktail of pro-inflammatory cytokines $(\mathrm{TNF} \alpha / \mathrm{IL}-1 \beta)$ to induce an inflamed state. Then, Inflamed HDFs were treated with one of three different photonic treatments: Light (a non-coherent light between 400 and $520 \mathrm{~nm}$ ); FLE-Gel of FLE-Matrix (a mix of non-coherent light and FLE with a broad spectrum between 400 and $700 \mathrm{~nm}$ ). All Inflamed and treated cells were maintained in the inflammatory cocktail environment post-treatment for the duration of the experiment.

Thirty minutes post-treatments, confocal microscopy analysis showed mitochondrial fragmentation in Inflamed HDFs and Light treated cells. Likewise, Motori and colleagues have previously shown that pro-inflammatory stimuli produced localized changes in mitochondrial dynamics, favoring fission over fusion [57]. Indeed, the mitochondrial architecture of a cell results from movement, tethering, fusion and fission events. Owing to frequent fission and fusion, different shapes of mitochondria can be found within a cell, including small vesicles, short rods, and long reticular networks. Strong evidence has demonstrated that mitochondrial dynamics are important for cell viability, senescence, mitochondria health, bioenergetic function, quality control, and intracellular signaling. Shortening of mitochondria is a result of increased fission activity or decreased fusion activity, and is typical for states of reduced bioenergetic efficiency (increased respiratory leak) [58].

Fragmentation of mitochondria may also indicate activation of the autophagic removal of mitochondria by a process known as mitophagy. This process is described as a pro-survival mechanism employed as an early response to cell stress, since it removes damaged mitochondria. Contrarily, if unfavorable condition persists, mitophagy results in cell death. Changes in mitochondrial morphology also occur in the early step of the cell death mechanisms apoptosis and necrosis $[59,60]$. The progressive loss of mitochondrial network during exposure to inflammatory mediators affirms that chronic 
inflammation affects mitochondrial morphology and bioenergetic functions. Of note, even as early as 30-min post-treatment, FLE-treated cells were starting to progress toward a healthier state of mitochondria, with significant differences observed between Inflamed HDFs and FLE-treated HDFs in the number of mitochondria per cell and the individual mitochondrion volume.

A full recovery of mitochondrial network occurred by 24-h post-treatment with FLE, manifested by mitochondrial networks comparable to those of Healthy HDFs. A trend towards recovery was also observed with Light-treated cells (PBM), however, while there were no significant differences from Healthy HDFs for mitochondria morphology, only the individual mitochondrion volume was different between Inflamed and Light-treated HDFs. This improved biological effect of FLE compared with PBM has been previously observed [36,40]. While not clearly understood, a possible explanation is biological sensitivity to changes in the light properties that arise during generation of FLE, including polarity, micro-pulsations, or coherency. Of note, opsin proteins are known to be sensitive to light polarity, thus it is feasible that other endogenous chromophores, including CCO, may be as well [61]. This enhanced impact of FLE compared with Light treatment is clearly visible in the radial graphs (Figure 3), with FLE-treated HDFs demonstrating both a quicker and more potent recovery.

Interestingly, we may see a dose-response starting to occur between FLE-Gel and FLE-Matrix. At 30-min, both groups have almost identical mitochondrial dynamics, however, by $24 \mathrm{~h}$ it appears as if the FLE-Matrix may be shifting the mitochondria network balance further towards fusion, even beyond that of the Healthy HDFs. However, there is no statistical significance to this finding, so further studies would be needed to better understand any dose effects associated with FLE and its biological implication. It is known that processes associated with increased energy are characterized by mitochondrial elongation and by respiration coupled to ATP synthesis [58]. We can hypothesize that the absorption of low energy photons by CCO has led to a restoration of the mitochondrial network due to an increase in enzymatic activity and respiration, and therefore an increase in ATP production.

Gene expression analysis did not report great alterations in expression of genes encoding enzymes of respiratory chain. In Light-treated cells, compared to Inflamed HDFs, upregulation of COX4I2, COX8C and UCP1 genes was observed. Little is reported about the expression of these genes in the literature, and nothing about their expression during the inflammatory process. However, Suárez and workers linked the upregulation of COX4I2 gene to the increase in UCP1 expression in an obese animal model [62]. They demonstrated that the overexpression of the thermogenic factor UCP1 improves metabolic phenotype concurrent with mitochondrial biogenesis trough the COX4I2 gene overexpression. In Light-treated cells, the improvement in mitochondrial biogenesis due to the upregulation of CCO-coding genes could be the basis of the mitochondrial morphology recorded 24 $\mathrm{h}$ post-treatment. In fact, through an increase in the number of mitochondria, a restoration of the mitochondrial volume equal to healthy cells has been observed. Conversely, in Inflamed cells, where COX6C gene downregulation was recorded, drastic mitochondrial fragmentation and a decrease in whole mitochondrial volume were found.

It was demonstrated that TNF- $\alpha$ and IL-1 $\beta$ decrease ATP production by reducing the activity of complex I, as well as by reducing mitochondrial membrane potential and inducing mitochondrial DNA damage [63]. The damage and mutations of mitochondrial DNA lead to the synthesis of functionally impaired respiratory chain subunits, promoting increased ROS production. In Inflamed cells, the increased production of ROS was confirmed by the upregulation of SOD2, the main mitochondrial antioxidant enzyme. It has been reported that when the inflammatory signal through the NF-KB pathway is strong and extended, the SOD2 expression is upregulated to maintain ROS homeostasis. However, if the ROS level is not restrained, cell damage and death occur [64]. In Light-treated cells, the levels of ROS are controlled by UCP1 upregulation via a slight decline in mitochondrial membrane potential, however allowing the production of ATP [44]. Since UCP1 was recently found deregulated during inflammation [65], and essential to preserve mitochondrial structural integrity and function [66,67], we can speculate that Light treatment may improve the mitochondrial function and recover mitochondrial network by the upregulation of this protein. 
Instead, treatment with FLE-Matrix resulted in upregulation of UCP1 as well as CTP1B, which is involved in mitochondrial $\beta$-oxidation of lipids long-chain fatty acids. Both genes are linked to an increase in ATP production because UCP separates oxidative phosphorylation from ATP synthesis allowing the production of ATP, also in the presence of ROS. Instead CTP1 favors $\beta$-oxidation of lipids ensuring the production of ATP [51,52]. It has already been shown that an increase in ATP synthesis is connected with the mitochondrial elongation under different cell conditions [58]. Compared to Light treatment, FLE-Matrix increased the volume of whole mitochondrial network through an increase in the volume of single mitochondria, favoring mitochondrial elongation. Having found the highest mitochondrial network recovery in this condition, we can assume that the gain in ATP production by mitochondrial $\beta$-oxidation may help in restoration of mitochondrial dynamics.

Conversely, an increase in UCP1 and CTP1B expression was not found in FLE-Gel treated cells, but an increase in SLC25A31 gene expression was observed. This gene encodes a nucleotide transporter which imports ADP into the mitochondrial matrix, where it can be converted to ATP by ATP synthase, and then exports the newly synthesized ATP to the cytosol [68]. Therefore, this carrier protein plays an important role in spending the ATP on metabolic processes necessary for cell survival. Since both FLE-Gel and FLE-Matrix recover mitochondrial network, it can be hypothesized that upregulation of this mitochondrial carrier causes an increase in ATP production due to the activation of ATP synthase.

ATP is the cellular energy-carrying molecule essential for multiple cellular functions. Reduced energy levels threaten cellular homeostasis and integrity. Impaired energy metabolism may trigger pro-apoptotic signaling, oxidative damage, cytotoxicity and impede mitochondrial DNA repair [69]. All the evaluated treatments led to an improvement in intracellular ATP levels, thus energizing the cells. However, it seems that FLE treatment rescued inflamed mitochondrial networks, returning them to a healthy condition despite the presence of oxidative stress caused by continued exposure to TNF $\alpha /$ IL-1 $\beta$ inflammatory cytokines. This return of mitochondrial homeostasis by FLE could be a key mechanism supporting accelerated healing and tissue regeneration in wounds and skin pathologies treated with FLE.

\section{Conclusions}

In conclusion, this work investigated the effect of FLE on mitochondrial homeostasis in an in vitro model of inflammation. FLE treatment leads to restoration of the mitochondrial network by 24-h post-treatment, as well as upregulation of $U C P 1$ and $C P T 1 B$ genes, which encode proteins favoring the production of ATP through oxidative phosphorylation and lipid $\beta$-oxidation, respectively. These observations, coupled with the previously established capacity of PBM to stimulate CCO, show a beneficial effect of FLE in the treatment of inflamed wounds. However, these findings derive from an in vitro model and should be validated by an in vivo investigation.

Author Contributions: Conceptualization, M.Z., S.E.C., L.H. and B.Z.; methodology, M.Z., L.F. and S.P.; formal analysis, M.Z., L.H., L.F. and S.P.; resources, M.Z., B.Z. and P.P.; data curation, L.F., M.Z., S.E.C and S.P.; writing-original draft preparation, M.Z., L.F. and S.P.; writing-review and editing, M.N., S.E.C, L.H., L.F. and S.P.; visualization, M.Z., C.S. and F.B.; supervision, B.Z. and P.P.; project administration, M.Z., S.E.C. and L.H. All authors have read and agreed to the published version of the manuscript.

Funding: This research was funded in part by Klox Technologies, Inc. Additionally, P.P. is supported by the Italian Association for Cancer Research (AIRC, IG-18624), Telethon (GGP11139B) and local funds from the University of Ferrara.

Acknowledgments: The authors would like to acknowledge Emanuela Buonamici for her technical and graphical support.

Conflicts of Interest: Drs. Zago, Campbell, Hébert, and Nielsen are employees of Klox Technologies. The other authors have no conflict of interest relevant to the content of this article. 


\section{References}

1. Gauglitz, G.G.; Korting, H.C.; Pavicic, T.; Ruzicka, T.; Jeschke, M.G. Hypertrophic scarring and keloids: Pathomechanisms and current and emerging treatment strategies. Mol. Med. 2011, 17, 113-125. [CrossRef] [PubMed]

2. Yager, D.R.; Chen, S.M.; Ward, S.I.; Olutoye, O.O.; Diegelmann, R.F.; Cohen, I.K. Ability of chronic wound fluids to degrade peptide growth factors is associated with increased levels of elastase activity and diminished levels of proteinase inhibitors. Wound Rep. Regen. 1997, 5, 23-32. [CrossRef] [PubMed]

3. Su, W.H.; Cheng, M.H.; Lee, W.L.; Tsou, T.S.; Chang, W.H.; Chen, C.S.; Wang, P.H. Nonsteroidal anti-inflammatory drugs for wounds: Pain relief or excessive scar formation? Mediat. Inflamm. 2010, 2010, 413238. [CrossRef] [PubMed]

4. Wang, J.; Wan, R.; Mo, Y.; Li, M.; Zhang, Q.; Chien, S. Intracellular delivery of adenosine triphosphate enhanced healing process in full-thickness skin wounds in diabetic rabbits. Am. J. Surg. 2010, 199, 823-832. [CrossRef] [PubMed]

5. Sen, C.K. Wound healing essentials: Let there be oxygen. Wound Repair. Regen. 2009, 17, 1-18. [CrossRef] [PubMed]

6. Gupta, A.; Raghubir, R. Energy metabolism in the granulation tissue of diabetic rats during cutaneous wound healing. Mol. Cell Biochem. 2005, 270, 71-77. [CrossRef]

7. Osellame, L.D.; Blacker, T.S.; Duchen, M.R. Cellular and molecular mechanisms of mitochondrial function. Best Pract. Res. Clin. Endocrinol. Metab. 2012, 26, 711-723. [CrossRef]

8. Bonora, M.; Patergnani, S.; Rimessi, A.; De Marchi, E.; Suski, J.M.; Bononi, A.; Giorgi, C.; Marchi, S.; Missiroli, S.; Poletti, F.; et al. ATP synthesis and storage. Purinergic. Signal 2012, 8, 343-357. [CrossRef]

9. Marchi, S.; Giorgi, C.; Suski, J.M.; Agnoletto, C.; Bononi, A.; Bonora, M.; De Marchi, E.; Missiroli, S.; Patergnani, S.; Poletti, F.; et al. Mitochondria-ros crosstalk in the control of cell death and aging. J. Signal Transduct. 2012, 2012, 329635. [CrossRef]

10. Landén, N.X.; Li, D.; Ståhle, M. Transition from inflammation to proliferation: A critical step during wound healing. Cell Mol. Life Sci. 2016, 73, 3861-3885. [CrossRef]

11. Tan, B.L.; Norhaizan, M.E.; Liew, W.P.; Sulaiman Rahman, H. Antioxidant and Oxidative Stress: A Mutual Interplay in Age-Related Diseases. Front Pharmacol. 2018, 9, 1162. [CrossRef] [PubMed]

12. Fujiwara, T.; Duscher, D.; Rustad, K.C.; Kosaraju, R.; Rodrigues, M.; Whittam, A.J.; Januszyk, M.; Maan, Z.N.; Gurtner, G.C. Extracellular superoxide dismutase deficiency impairs wound healing in advanced age by reducing neovascularization and fibroblast function. Exp. Dermatol. 2016, 25, 206-211. [CrossRef] [PubMed]

13. Jiang, F.; Zhang, Y.; Dusting, G.J. NADPH oxidase-mediated redox signalling: Roles in cellular stress response, stress tolerance and tissue repair. Pharmacol. Rev. 2011, 63, 218-242. [CrossRef] [PubMed]

14. Murphy, M.P. Modulating mitochondrial intracellular location as a redox signal. Sci Signal. 2012, 5, pe39. [CrossRef]

15. Haga, N.; Fujita, N.; Tsuruo, T. Mitochondrial aggregation precedes cytochrome c release from mitochondria during apoptosis. Oncogene 2003, 22, 5579-5585. [CrossRef]

16. Nakada, K.; Inoue, K.; Ono, T.; Isobe, K.; Ogura, A.; Goto, Y.I.; Nonaka, I.; Hayashi, J.I. Inter-mitochondrial complementation: Mitochondria-specific system preventing mice from expression of disease phenotypes by mutant mtDNA. Nat. Med. 2001, 7, 934-940. [CrossRef]

17. Karbowski, M.; Youle, R.J. Dynamics of mitochondrial morphology in healthy cells and during apoptosis. Cell Death Differ. 2003, 10, 870-880. [CrossRef] [PubMed]

18. Desai, S.P.; Bhatia, S.N.; Toner, M.; Irimia, D. Mitochondrial Localization and the Persistent Migration of Epithelial Cancer cells. Biophys. J. 2013, 104, 2077-2088. [CrossRef] [PubMed]

19. Boldogh, I.R.; Pon, L.A. Interactions of mitochondria with the actin cytoskeleton. Biochim. Biophys. Acta 2006, 1763, 450-462. [CrossRef] [PubMed]

20. Avci, P.; Gupta, A.; Sadasivam, M.; Vecchio, D.; Pam, Z.; Pam, N.; Hamblin, M.R. Low level laser (light) Therapy (LLLT) in skin: Stimulating, healing, restoring. Semin. Cutan. Med. Surg. 2013, 32, 42-52.

21. Anders, J.J.; Lanzafme, R.J.; Arany, P.R. Low level light/laser therapy versus Photobiomodulation therapy. Photomed. Laser Surg. 2015, 33, 183-184. [CrossRef] [PubMed]

22. Hamblin, M.R. Photobiomodulation or low-level laser therapy. J. Biophotonics. 2016, 9, 1122-1124. [CrossRef] [PubMed] 
23. Enwemeka, C.S.; Parker, J.C.; Dowdy, D.S.; Harkness, E.E.; Sanford, L.E.; Woodruff, L.D. The efficacy of low-power lasers in tissue repair and pain control: A meta-analysis study. Photomed. Laser Surg. 2004, 22, 323-329. [CrossRef] [PubMed]

24. Woodruff, L.D.; Bounkeo, J.M.; Brannon, W.M.; Dawes, K.S.; Barham, C.D.; Waddell, D.L.; Enwemeka, C.S. The efficacy of laser therapy in wound repair: A meta-analysis of the literature. Photomed. Laser Surg. 2004, 22, 241-247. [CrossRef] [PubMed]

25. Traverzim, M.; Makbe, S.; Sivla, D.F.T.; Pavani, C.1; Bussadori, S.K.; Fernandes, K.S.P.; Motta, L.J. Effect of LED photobiomodulation on analgesia during labour: Study protocol for randomized control clinical trial. Medicine 2018, 97, e11120. [CrossRef]

26. Da-Palma-Cruz, M.; da Silva, R.F.; Monteiro, D.; Rehim, H.M.M.A.; Grabulosa, C.C.; de Oliveira, A.P.L.; Lino-Dos-Santos-Franco, A. Photobiomodulation modulates the resolution of inflammation during acute lung injury induced by sepsis. Lasers. Med. Sci. 2019, 34, 191-199. [CrossRef]

27. Langella, L.G.; Casalechi, H.L.; Tomazoni, S.S.; Johnson, D.S.; Albertini, R.; Pallotta, R.C.; Marcos, R.L.; de Carvalho, P.T.C.; Leal-Junior, E.C.P. Photobiomodulation Therapy (PBMT) on acute pain and inflammation in patients who underwent total hip arthroplasty- a randomized, triple blind, placebo controlled clinical trial. Lasers. Med. Sci. 2018, 33, 1933-1940. [CrossRef]

28. Liebert, A.; Krause, A.; Goonetilleke, N.; Bicknell, B.; Kiat, H. A Role for Photobiomodulation in the Prevention of Myocardial Ischemic Reperfusion Injury: A Systematic Review and Potential Molecular Mechanisms. Sci. Rep. 2017, 7, 42386. [CrossRef]

29. Hamblin, M.R. Photobiomodulation for traumatic brain injury and stroke. J. Neurosci. Res. 2018, 96, 731-743. [CrossRef]

30. Mosca, R.C.; Ong, A.A.; Albasha, O.; Bass, K.; Arany, P. Photobiomodulation Therapy for Wound Care: A Potent, Noninvasive, Photoceutical Approach. Adv. Skin Wound Care 2019, 32, 157-167. [CrossRef]

31. Karu, T.I.; Afanas'eva, N.I. Cytochrome c oxidase as the primary photoacceptor upon laser exposure of cultured cells to visible and near IR-range light. Dokl. Akad. Nauk. 1995, 342, 693-695. [PubMed]

32. Karu, T.I.; Pyatibrat, L.V.; Kolyakov, S.F.; Afanasyeva, N.I. Absorption measurements of a cell monolayer relevant to phototherapy: Reduction of cytochrome C oxidase under near IR radiation. J. Photochem. Photobiol. B 2005, 81, 98-106. [CrossRef] [PubMed]

33. Amaroli, A.; Ravera, S.; Baldini, F.; Benedicenti, S.; Panfoli, I.; Vergani, L. Photobiomodulation with 808-nm diode laser light promotes wound healing of human endothelial cells through increased reactive oxygen species production stimulating mitochondrial oxidative phosphorylation. Lasers Med. Sci. 2019, 34, 495-504. [CrossRef] [PubMed]

34. Tatmatsu-Rocha, J.C.; Tim, C.R.; Avo, L.; Bernardes-Filho, R.; Brassolatti, P.; Kido, H.W.; Hamblin, M.R.; Parizotto, N.A. Mitochondrial dynamics (fission and fusion) and collagen production in a rat model of diabetic wound healing treated by photobiomodulation: Comparison of $904 \mathrm{~nm}$ laser and $850 \mathrm{~nm}$ light-emitting diode (LED). J. Photochem. Photobiol. B 2018, 187, 41-47. [CrossRef]

35. Korobova, F.; Ramabhadran, V.; Higgs, H.N. An actin-dependent step in mitochondrial fission mediated by the ER-associated formin INF2. Science 2013, 339, 464-467. [CrossRef]

36. Scapagnini, G.; Marchegiani, A.; Rossi, G.; Zago, M.; Jowarska, J.; Wael, M.; Campbell, S.E.; Schiffman, Z.; Buonamici, E.; Garvao, R.; et al. Management of all three phases of wound healing through the induction of fluorescence biomodulation using fluorescence light energy, Proc. SPIE 10863, Photonic Diagnosis and Treatment of Infections and Inflammatory Diseases II, 108630W, San Francisco, California, United States, 7 March 2019; International Society for Optics and Photonics: Bellingham, WA, USA, 2019; Available online: https://doi.org/10.1117/12.2508066 (accessed on 8 November 2019).

37. Romanelli, M.; Piaggesi, A.; Scapagnini, G.; Dini, V.; Janowska, A.; Iacopi, E.; Scarpa, C.; Fauverghe, S.; Bassetto, F.; EUREKA Study Group. Evaluation of fluorescence biomodulation in the real-life management of chronic wounds: The EURIKA trial. J. Wound Care 2018, 27, 744-753. [CrossRef]

38. Dini, V.; Janowska, A.; Davini, G.; Kerihuel, J.C.; Fauverghe, S.; Romanelli, M. Biomodulation induced by fluorescent light energy versus standard of care in venous leg ulcers: A retrospective study. J. Wound Care 2019, 28, 730-736. [CrossRef]

39. Nikolis, A.; Griard, D.; Pesant, Y.; Scapagnini, G.; Vezina, D. A prospective case series evaluating the safety and efficacy of the Klox Biophotonic System in venous leg ulcers. Chron. Wound Care Manag. Res. 2016, 3, 101-111. [CrossRef] 
40. Edge, D.; Mellergaard, M.; Dam-Hansen, C.; Corell, D.D.; Jaworska, J.; Scapagnini, G.; Nielsen, M.C.E. FLUORESCENT LIGHT ENERGY: The Future for Treating Inflammatory Skin Conditions? J. Clin. Aesthet. Dermatol. 2019, 12, E61-E68.

41. Koceva, I.; Rümmelein, B.; Gerber, P.A.; Edge, D.; Nielsen, M.C.E. Fluorescent light energy: A new therapeutic approach to effectively treating acne conglobata and hidradenitis suppurativa. Clin. Case Rep. 2019, 7, 1769-1772. [CrossRef]

42. Sannino, M.; Lodi, G.; Dethlefsen, M.W.; Nisticò, S.P.; Cannarozzo, G.; Nielsen, M.C.E. Fluorescent light energy: Treating rosacea subtypes 1, 2, and 3. Clin. Case Rep. 2018, 6, 2385-2390. [CrossRef] [PubMed]

43. Marchegiani, A.; Spaterna, A.; Cerquetella, M.; Tambella, A.M.; Fruganti, A.; Paterson, S. Fluorescence biomodulation in the management of canine interdigital pyoderma cases: A prospective, single-blinded, randomized and controlled clinical study. Vet. Dermatol. 2019, 30, 371-e109. [CrossRef] [PubMed]

44. Ferroni, L.; Gardin, C.; Dalla Paola, L.; Campo, G.; Cimaglia, P.; Bellin, G.; Pinton, P.; Zavan, B. Characterization of Dermal Stem Cells of Diabetic Patients. Cells 2019, 8, 729. [CrossRef] [PubMed]

45. Rutter, J.; Winge, D.R.; Schiffman, J.D. Succinate dehydrogenase-Assembly, regulation and role in human disease. Mitochondrion 2010, 10, 393-401. [CrossRef] [PubMed]

46. Gutiérrez-Aguilar, M.; Baines, C.P. Physiological and pathological roles of mitochondrial SLC25 carriers. Biochem. J. 2013, 454, 371-386. [CrossRef]

47. Medina, A.; Ghaffari, A.; Kilani, R.T.; Ghahary, A. The role of stratifin in fibroblast-keratinocyte interaction. Mol. Cell Biochem. 2007, 305, 255-264. [CrossRef]

48. Agarwal, P.; Sandey, M.; DeInnocentes, P.; Bird, R.C. Tumor suppressor gene p16/INK4A/CDKN2A-dependent regulation into and out of the cell cycle in a spontaneous canine model of breast cancer. J. Cell. Biochem. 2013, 114, 1355-1363. [CrossRef]

49. Han, J.; Flemington, C.; Houghton, A.B.; Gu, Z.; Zambetti, G.P.; Lutz, R.J.; Zhu, L.; Chittenden, T. Expression of bbc3, a pro-apoptotic BH3-only gene, is regulated by diverse cell death and survival signals. Proc. Natl. Acad. Sci. USA 2001, 98, 11318-11323. [CrossRef]

50. Amaral, J.D.; Xavier, J.M.; Steer, C.J.; Rodrigues, C.M. The role of p53 in apoptosis. Discov. Med. 2010, 9, 145-152.

51. Zhao, R.Z.; Jiang, S.; Zhang, L.; Yu, Z.B. Mitochondrial electron transport chain, ROS generation and uncoupling. Int. J. Mol. Med. 2019, 44, 3-15. [CrossRef]

52. Longo, N.; Frigeni, M.; Pasquali, M. Carnitine transport and fatty acid oxidation. Biochim. Biophys. Acta 2016, 1863, 2422-2435. [CrossRef] [PubMed]

53. Palmieri, F. The mitochondrial transporter family SLC25: Identification, properties and physiopathology. Mol. Aspects. Med. 2013, 34, 465-484. [CrossRef] [PubMed]

54. Chaban, Y.; Boekema, E.J.; Dudkina, N.V. Structures of mitochondrial oxidative phosphorylation supercomplexes and mechanisms for their stabilisation. Biochim. Biophys. Acta 2014, 1837, 418-426. [CrossRef] [PubMed]

55. Passarella, S.; Karu, T. Absorption of monochromatic and narrow band radiation in the visible and near IR by both mitochondrial and non-mitochondrial photoacceptors results in photobiomodulation. J. Photochem. Photobiol. B 2014, 140, 344-358. [CrossRef]

56. Pannala, V.R.; Camara, A.K.; Dash, R.K. Modeling the detailed kinetics of mitochondrial cytochrome c oxidase: Catalytic mechanism and nitric oxide inhibition. J. Appl. Physiol. 2016, 121, 1196-1207. [CrossRef]

57. Motori, E.; Puyal, J.; Toni, N.; Ghanem, A.; Angeloni, C.; Malaguti, M.; Cantelli-Forti, G.; Berninger, B.; Conzelmann, K.K.; Götz, M.; et al. Inflammation-induced alteration of astrocyte mitochondrial dynamics requires autophagy for mitochondrial network maintenance. Cell Metab. 2013, 18, 844-859. [CrossRef]

58. Liesa, M.; Shirihai, O.S. Mitochondrial dynamics in the regulation of nutrient utilization and energy expenditure. Cell Metab. 2013, 17, 491-506. [CrossRef]

59. Chipuk, J.E.; Moldoveanu, T.; Llambi, F.; Parsons, M.J.; Green, D.R. The BCL-2 family reunion. Mol. Cell. 2010, 12, 299-310. [CrossRef]

60. Zong, W.X.; Thompson, C.B. Necrotic death as a cell fate. Genes. Dev. 2006, 1, 1-15. [CrossRef]

61. Hardie, C.R. Polarization Vision: Drosophila Enters the Arena. Curr. Biol. 2012, 22, R12-R14. [CrossRef] 
62. Suárez, J.; Rivera, P.; Arrabal, S.; Crespillo, A.; Serrano, A.; Baixeras, E.; Pavón, F.J.; Cifuentes, M.; Nogueiras, R.; Ballesteros, J.; et al. Oleoylethanolamide enhances $\beta$-adrenergic-mediated thermogenesis and white-to-brown adipocyte phenotype in epididymal white adipose tissue in rat. Dis. Model Mech. 2014, 7, 129-141. [CrossRef] [PubMed]

63. Hosseinzadeh, A.; Kamrava, S.K.; Joghataei, M.T.; Darabi, R.; Shakeri-Zadeh, A.; Shahriari, M.; Reiter, R.J.; Ghaznavi, H.; Mehrzadi, S. Apoptosis signaling pathways in osteoarthritis and possible protective role of melatonin. J. Pineal Res. 2016, 61, 411-425. [CrossRef] [PubMed]

64. Yoon, Y.; Kim, T.J.; Lee, J.M.; Kim, D.Y. SOD2 is upregulated in periodontitis to reduce further inflammation progression. Oral. Dis. 2018, 24, 1572-1580. [CrossRef] [PubMed]

65. Nøhr, M.K.; Bobba, N.; Richelsen, B.; Lund, S.; Pedersen, S.B. Inflammation Downregulates UCP1 Expression in Brown Adipocytes Potentially via SIRT1 and DBC1 Interaction. Int. J. Mol. Sci. 2017, 18, 1006. [CrossRef]

66. Lee, J.Y.; Takahashi, N.; Yasubuchi, M.; Kim, Y.I.; Hashizaki, H.; Kim, M.J.; Sakamoto, T.; Goto, T.; Kawada, T. Triiodothyronine induces UCP-1 expression and mitochondrial biogenesis in human adipocytes. Am. J. Physiol. Cell Physiol. 2012, 302, C463-C472. [CrossRef]

67. Ortega, S.P.; Chouchani, E.T.; Boudina, S. Stress turns on the heat: Regulation of mitochondrial biogenesis and UCP1 by ROS in adipocytes. Adipocyte 2017, 6, 56-61. [CrossRef]

68. Ruprecht, J.J.; Kunji, E.R.S. The SLC25 Mitochondrial Carrier Family: Structure and Mechanism. Trends Biochem. Sci. 2019, 45, 224-258. [CrossRef]

69. Owen, L.; Sunram-Lea, S.I. Metabolic agents that enhance ATP can improve cognitive functioning: A review of the evidence for glucose, oxygen, pyruvate, creatine, and L-carnitine. Nutrients 2011, 3, 735-755. [CrossRef]

(C) 2020 by the authors. Licensee MDPI, Basel, Switzerland. This article is an open access article distributed under the terms and conditions of the Creative Commons Attribution (CC BY) license (http://creativecommons.org/licenses/by/4.0/). 